\title{
Discovery of Bis-sydnone styryl ketone as a selective cyclooxygenase-2 inhibitor
}

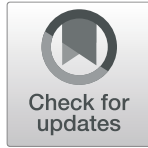

Abdualrahman Mohammed Abdualkader ${ }^{1 *}$ (D, Muhammad Taher $^{2}$, Nik Idris Nik Yusoff ${ }^{1}$ and Mohamed Alaama ${ }^{1}$

\begin{abstract}
Background: Various literature sources have documented a wide spectrum of therapeutic properties of sydnones including anti-inflammatory, anticancer, antimicrobial activities. Phenyl styryl ketones and their derivatives as members of the chalcone family have also been reported as significant bioactive molecules. The current study was initiated to evaluate the anti-inflammatory activity of sydnone-based compounds including some novel bis-sydnone styryl ketone hybrids.

Results: Twenty-five sydnone-containing compounds were successfully synthesized. Compounds $46-48$ and 56-58 were reported as new sydnone derivatives. Whereas, compounds 61-63 were synthesized as novel molecules containing two sydnone rings linked via $\alpha, \beta$-unsaturated ketone. The structures of the synthesized compounds were confirmed by FTIR, ${ }^{1} \mathrm{H}$ NMR, ${ }^{13} \mathrm{C}$ NMR and ToF-MS analyses. The in vitro COX inhibition assay showed varied activity. Compounds 47, 51, 58 and 63 showed the most potent COX inhibitory effects at a concentration of 200 $\mu \mathrm{M}$. The selectivity index revealed that 63 was the best selective COX-2 inhibitor. Acetylation of the sydnone ring at C-4 was fruitful for the COX inhibitory effects. Docking analysis showed that COX-2 selectivity was due to a favourable positive charged interaction occurring between the sydnone ring of 63 and Arg513 of COX-2. Compound 51 was hydrogen bonded to Arg513. On the other hand, the low inhibitory effect of 63 against COX-1 was due to an unfavourable polar interaction with His513 in the binding pocket of COX-1.

Conclusions: The compounds were successfully synthesized and characterized. Compound 63 had a common architecture and pharmacophoric features with known selective COX-2 inhibitors (the coxib family) which make it a suitable candidate for the designing of selective and safe NSAID.
\end{abstract}

Keywords: Sydnone, Heterocycle, Mesoionic, Cyclooxygenase, Anti-inflammatory

\section{Background}

Sydnone can be defined as a five-membered pseudoaromatic heterocyclic molecule. Oxadiazoles, classically 1,2,3-oxadiazole, form the main skeleton for sydnone (Fig. 1). The molecule has delocalized balanced positive and negative charges. The positive charge is shared between the five annular atoms, but mainly located at the nitrogen atom N3. An enolate-like exocyclic oxygen atom bears the negative charge. Studies on sydnone structure showed that there is no single polar or

\footnotetext{
* Correspondence: armak1983@gmail.com

'Pharmaceutical Chemistry Department, Faculty of Pharmacy, International

Islamic University Malaysia, 25200 Kuantan, Pahang, Malaysia

Full list of author information is available at the end of the article
}

covalent structure able to represent the sydnone ring satisfactorily. The hydrogen atom at the position $\mathrm{C} 4$ was demonstrated to have acidic and nucleophilic functionalities making the sydnone ring reactive towards electrophilic reagents [1].

The history of sydnone dates back to the early nineteenth century when Earl and his colleagues reported the formation of the sydnone ring as the result of the effect of acetic anhydride on $N$-nitroso amino acids $[1,2]$. It was well documented that sydnone-containing compounds have a broad spectrum of biological activities including antitumor, antidiabetic, antimicrobial, antioxidant and anti-inflammatory [3]. The exploration of the bioactivity of sydnones appended to other 


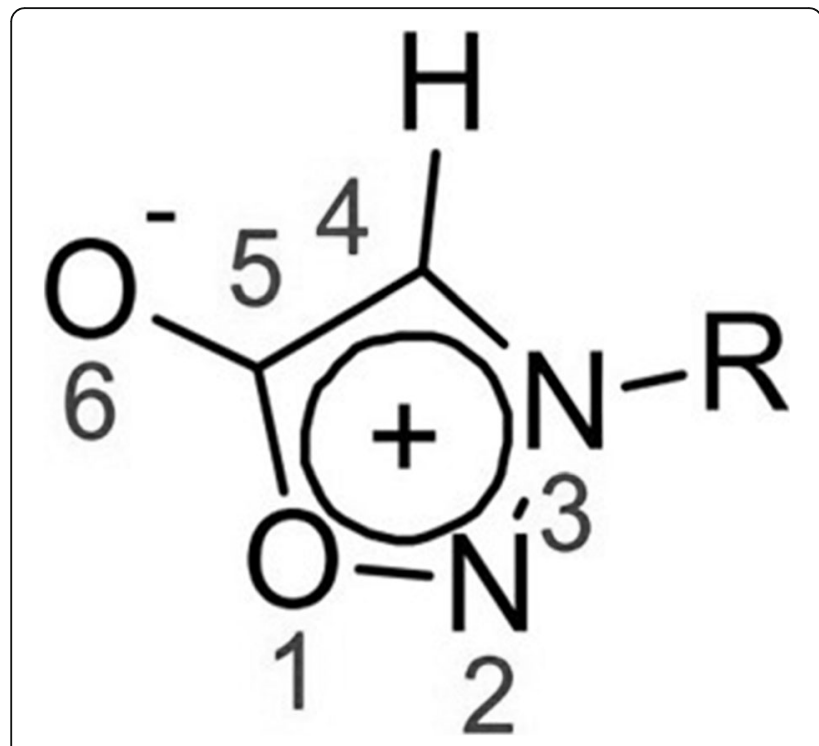

Fig. 1 Sydnone structure

molecules was explored. Structure-activity relationship (SAR) studies were mainly directed towards the adjunct structures like thiazole, benzothiazole, pyrimidine and sulphonamide overlooking the unique sydnone ring [4, 5]. The anti-inflammatory activity of sydnones was initially published by Wagner and Hill who reported 3-(2arylthioethyl)sydnone and 3-(2-arylsulfoxyethyl)sydnone as efficacious anti-edema agents in rats $[6,7]$.

Considering these issues, we undertook this research to cover the lack of consistent SAR data on activity variations resulted from structural modification on the sydnone ring itself. We hypothesize that joining two sydnone rings through a phenyl styrylketone linker will produce selective cyclooxygenase-2 (COX-2) inhibitors by giving the new molecules common architecture and pharmacophoric features with known selective COX-2 inhibitors (coxib family). We hypothesize that the nucleophilic hydrogen atom at $\mathrm{C} 4$ of the sydnone ring, the various substituents to the phenyl ring at N3, and connecting two sydnone rings via a phenyl styrylketone linker have a crucial impact on the COX-2 inhibitory activity of sydnone-based compounds.

\section{Methods}

\section{Chemical synthesis}

All chemicals and solvents were purchased from commercial suppliers and used without purification. Reactions were monitored using thin-layer chromatography (TLC) performed on Silica Gel $60 \mathrm{~F}_{254}$ plates (Merck) using a suitable mobile phase system. Column chromatography was carried out on silica gel (60-200 mesh) supplied from Merck. Melting points $\left(\right.$ Celsius, $\left.{ }^{\circ} \mathrm{C}\right)$ were uncorrected and determined using open-end capillary tubes on Stuart Scientific apparatus. Infrared spectra
(IR) were obtained via potassium bromide (Merck, IR grade) discs using Perkin-Elmer Spectrum Two FT-IR instrument. ${ }^{1} \mathrm{H}-\mathrm{NMR}(700 \mathrm{MHz})$ and ${ }^{13} \mathrm{C}-\mathrm{NMR}$ (76 $\mathrm{MHz}$ ) spectra were recorded at $298 \mathrm{~K}$ on a Bruker Ascend spectrometer equipped with $5 \mathrm{~mm}$ TCI inverse detection cryoprobe. Chemical shifts were recorded in parts per million (ppm, $\delta$ ) relative to tetramethylsilane (TMS) as an internal standard. High-resolution mass spectrometry (HRMS) was conducted using electrospray ionization techniques (ESI) by Bruker micrOTOF-Q II.

\section{Synthesis of ethyl $\mathrm{N}$-arylglycinate esters 6-10}

As shown in Fig. 2, a mixture of the corresponding para substituted aniline 1-5 (0.1 mol), ethyl chloroacetate $(0.2 \mathrm{~mol})$ and anhydrous sodium acetate $(0.2 \mathrm{~mol})$ were refluxed for $12 \mathrm{~h}$ in $100 \mathrm{~mL}$ absolute ethanol. The mixture was kept in the fridge overnight after adding 100 $\mathrm{mL}$ hot water [8]. The precipitated solid was vacuumfiltered and then recrystallised from $95 \%$ ethanol to produce $N$-arylglycine esters 6-10.

\section{Synthesis of methyl 4-aminobenzoate 12}

The general protocol of Fisher's esterification was followed to prepare methyl 4-aminobenzoate 12 (Fig. 2). In $50 \mathrm{~mL}(1.26 \mathrm{~mol})$ hot methanol, $0.105 \mathrm{~mol}$ paraamino benzoic acid $\mathbf{1 1}$ was dissolved. Concentrated sulphuric acid $(2.50 \mathrm{~mol})$ was added dropwise with continuous cooling. The resulting white paste was heated at $90-95{ }^{\circ} \mathrm{C}$ for $1 \mathrm{~h}$. The reaction mixture was cooled down to ambient room temperature, then neutralised with an aqueous solution of sodium carbonate $5 \% \mathrm{w} / \mathrm{v}$. The white solid was then collected and recrystallised from ethanol.

\section{Synthesis of N-arylglycine 13-17}

As described by Patel and his colleage [8], the prepared esters 6-10 $(0.02 \mathrm{~mol})$ and sodium hydroxide $(0.04 \mathrm{~mol})$ were heated at $80-85{ }^{\circ} \mathrm{C}$ in a mixture of water and ethanol $(36: 8 \mathrm{~mL})$ for $1 \mathrm{~h}$. After cooling to ambient room temperature, the reaction mixture was acidified by fuming hydrochloric acid in a dropwise manner to precipitate the corresponding amino acids 13-17 (Fig. 2) which were collected via filtration and used for the next step without further purification.

\section{Synthesis of nitro-containing $\mathrm{N}$-arylglycine 22-25}

An aqueous solution of chloroacetic acid $(0.2 \mathrm{~mol})$ was neutralised with an equimolar of $\mathrm{NaOH}$ and then added to a boiling suspension of nitro-containing aniline derivatives 18-21 (0.1 mol) over a period of $6 \mathrm{~h}$ (Fig. 2). Reflux was continued for another $72 \mathrm{~h}$ with stirring. The black residue was then removed by vacuum filtration while hot, and the filtrate was kept in the fridge overnight. The resulting yellow solid was collected and 


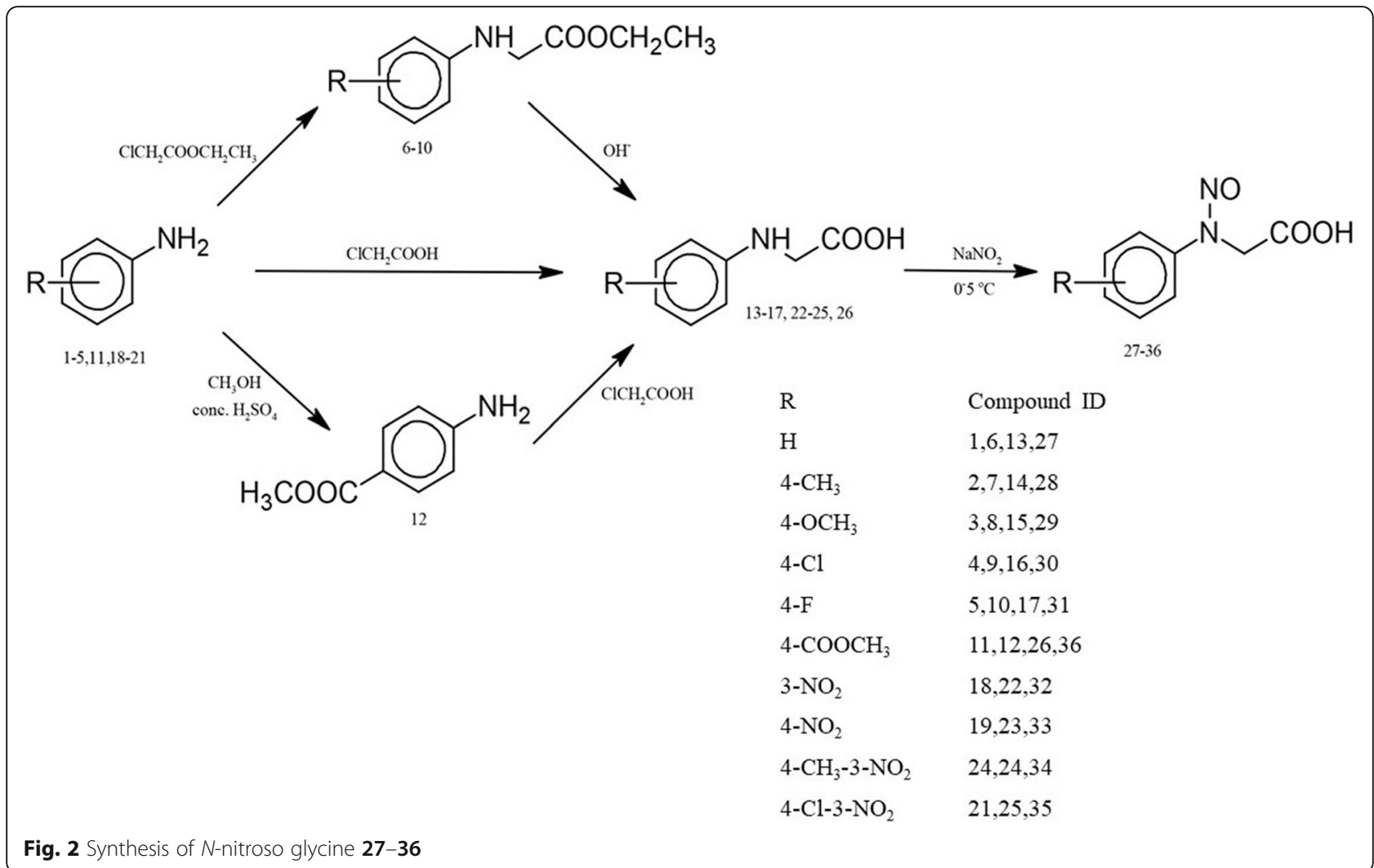

gently refluxed with ammonia $(20 \% \mathrm{v} / \mathrm{v})$ for $30 \mathrm{~min}$. After cooling to room temperature, the unreacted initiating material was removed via filtration. The filtrate was acidified with concentrated hydrochloric acid [9]. The yellow product was vacuum-filtered and recrystallised from ethanol to yield the corresponding amino acids 22-25.

\section{Synthesis of N-[4-(methoxycarbonyl)phenyl]glycine 26}

A mixture of $12(0.15 \mathrm{~mol})$, chloroacetic acid $(0.1 \mathrm{~mol})$ and sodium acetate trihydrate $(0.1 \mathrm{~mol})$ in $100 \mathrm{~mL}$ distilled water was refluxed for $6 \mathrm{~h}$ (Fig. 2). Sodium hydroxide $(10 \% \mathrm{w} / \mathrm{v})$ was next used to bring the $\mathrm{pH}$ to 8.0 . Following extraction by $3 \times 100 \mathrm{~mL}$ dichloromethane, the product was precipitated by acidifying the aqueous layer using concentrated hydrochloric acid [10]. Recrystallisation from ethanol produced $N$-[4-(methoxycarbonyl)phenyl] glycine $\mathbf{2 6}$.

\section{Synthesis of $\mathrm{N}$-nitroso- $\mathrm{N}$-arylglycine 27-36}

To a suspension of the prepared $N$-arylglycine $(0.04$ mol) in $100 \mathrm{~mL}$ cold water, one drop of concentrated hydrochloric acid was added. In $20 \mathrm{~mL}$ of cold water, 0.12 mol sodium nitrite was dissolved and added dropwise with vigorous stirring at $0-5{ }^{\circ} \mathrm{C}$ over a period of 1 h (Fig. 2). Stirring at ambient room temperature continued for $0.5-4 \mathrm{~h}$ until a clear liquor solution was achieved. The concentrated hydrochloric acid was then added slowly, and the precipitated nitroso derivatives were vacuum-filtered [8]. The products 27-36 were used for the following steps without further purification.

\section{Synthesis of 3-arylsydnones 37-46}

In $15 \mathrm{~mL}$ dichloromethane, $0.015 \mathrm{~mol}$ of the prepared nitroso compounds $27-36$ and $0.022 \mathrm{~mol}$ of trifluoroacetic anhydride (TFAA) were stirred at $0-5{ }^{\circ} \mathrm{C}$ until complete dissolution as shown in Fig. 3 [11]. Next, the dichloromethane was evaporated under a nitrogen stream, and the resultant pink solid was washed with 5 $\mathrm{mL}$ sodium carbonate $(10 \% \mathrm{w} / \mathrm{v})$ and then recrystallised from ethanol $95 \%$ to produce the corresponding sydnones 37-46.

\section{Synthesis of alcohol sydnone derivatives 47}

A mixture of sydnone $46(0.018 \mathrm{~mol})$ and sodium borohydride $(0.052 \mathrm{~mol})$ was heated until reflux in $190 \mathrm{~mL}$ of tert-butanol (Fig. 3). Then, $20 \mathrm{~mL}$ methanol was added dropwise for $30 \mathrm{~min}$ with reflux continuing for a further $2 \mathrm{~h}$. After cooling to room temperature, $200 \mathrm{~mL}$ water was added. Fractional distillation evaporated the remaining methanol and $t$-butanol as azeotrope mixture [10]. The remaining liquor aqueous solution was kept in the fridge overnight to precipitate the alcohol sydnone derivative as a dark red solid which was recrystallised 


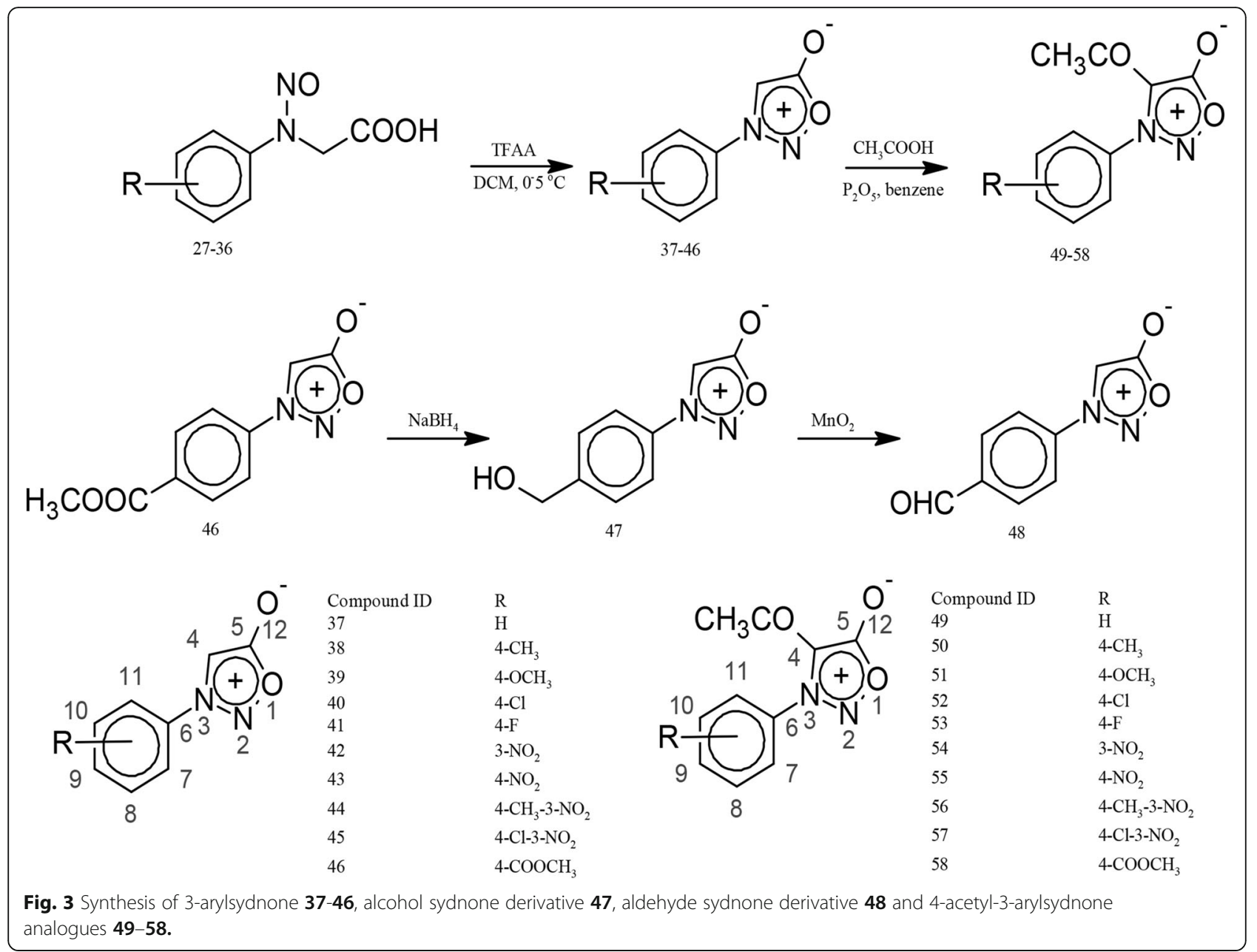

from ethanol to produce 3-[4-(Hydroxymethyl)phenyl]sydnone 47.

\section{Synthesis of aldehyde sydnone derivatives 48}

A suspension of $47(0.02 \mathrm{~mol})$ and $\mathrm{MnO}_{2}(0.14 \mathrm{~mol})$ in $360 \mathrm{~mL}$ dichloromethane was stirred at room temperature for $12 \mathrm{~h}$ (Fig. 3). The reaction mixture was then filtered to remove the remaining starting materials [10]. Dichloromethane was evaporated under reduced pressure, and the resulting crude product was recrystallised from ethyl acetate to afford 3-(4-formylphenyl)sydnone 48.

\section{Synthesis of 4-acetyl-3-arylsydnones 49-58}

In $20 \mathrm{~mL}$ of dry benzene $0.00375 \mathrm{~mol}$ of sydnones $37-$ 48 and $0.0105 \mathrm{~mol}$ of phosphorous pentoxide were heated until reflux. Then, $0.600 \mathrm{~mL}$ glacial acetic acid was slowly added during the next $20 \mathrm{~min}$ with vigorous stirring (Fig. 3). The reaction mixture was then refluxed for a further $5 \mathrm{~h}$ under a nitrogen condition. The benzene layer was decanted, and the black residue was washed with $3 \times 20 \mathrm{~mL}$ hot benzene. The combined benzene extracts were dried to produce the crude 4acetylated sydnone [12]. Compounds 49-53 were purified by recrystallisation from warm ethanol $\left(60{ }^{\circ} \mathrm{C}\right)$. Compounds 54-58 were eluted through a column of silica gel $60(0.063-0.200 \mathrm{~mm})$ using ethyl acetate and hexane $(1: 1)$ as a mobile phase to remove the unreacted initiating materials, followed by recrystallisation from boiling ethanol. Compounds 59 bearing $-\mathrm{CH}_{2} \mathrm{OH}$ at the para position of the benzene ring (corresponding to sydnone 47) and 60 bearing - $\mathrm{CHO}$ at the para position of the benzene ring (corresponding to sydnone 48) were not found.

\section{Synthesis of bis-sydnone styryl ketones 61-63}

A mixture of 4-acetyl-3-arylsydnone 49-58 (0.0025 mol) and sodium hydroxide $(0.00375 \mathrm{~mol})$ was dissolved in water $(0.75 \mathrm{~mL})$ and ethanol $(5 \mathrm{~mL})$, and then stirred for $1 \mathrm{~h}$ at $5{ }^{\circ} \mathrm{C}$. Then, 3-(4-formylphenyl)sydnone 48 (0.003 mol) was added portion-wise with vigorous stirring within 30 min (Fig. 4). The reaction mixture was stirred for another hour on an ice bath until complete dissolution, followed by $12 \mathrm{~h}$ at room temperature [13]. The 


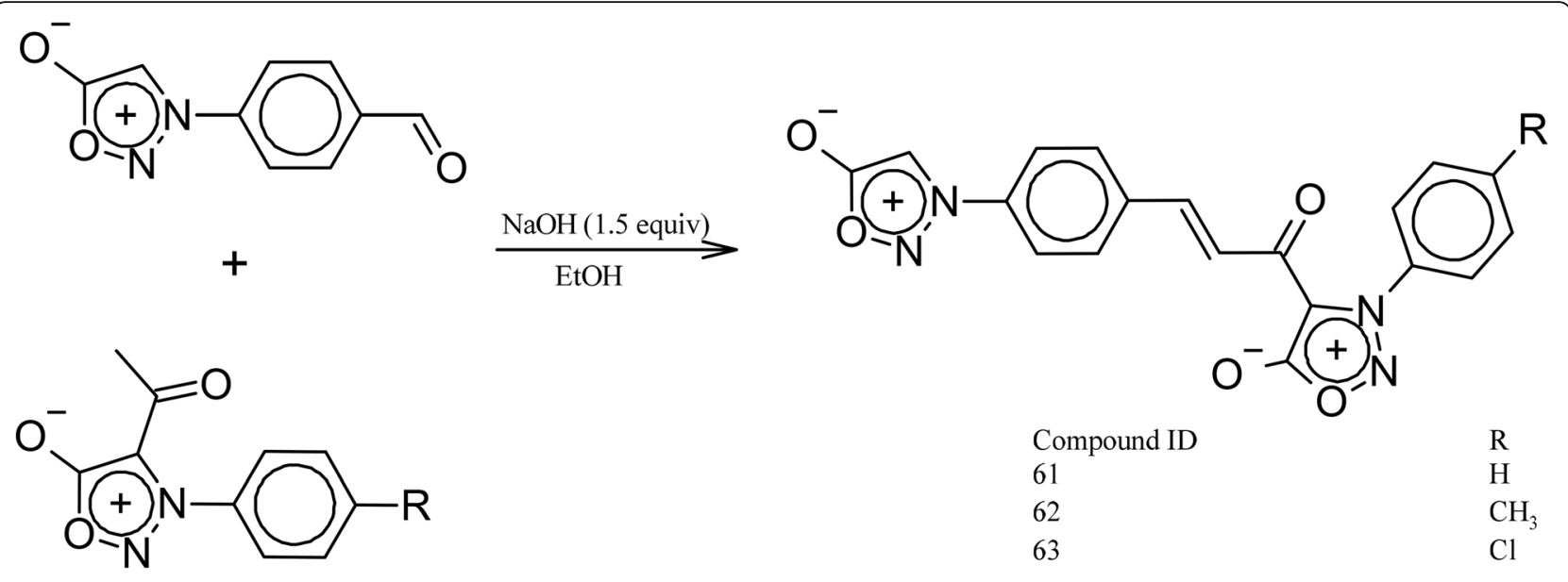

Fig. 4 Synthesis of bis-sydnone styryl ketone 61-63

deep yellow precipitate was filtered, washed thoroughly with cold water and dried in a desiccator. Compounds 61 and 62 were purified by recrystallisation from ethanol and ethyl acetate (1:1). Compound 63 was eluted through a column of silica gel $60(0.063-0.200 \mathrm{~mm})$ using dichloromethane and ethyl acetate (95:5) as mobile phase and then recrystallised from dioxane.

\section{Biological evaluation (in vitro cyclooxygenase inhibitory assay)}

The inhibitory activity of sydnones 37-58 and 61-63 (at a final concentration of $200 \mu \mathrm{M}$ ) against ovine COX-1 and human recombinant COX-2 was evaluated using COX (ovine/human) inhibitor screening assay kit (Catalogue No. 560131) from Cayman Chemicals, Michigan, USA following the procedure suggested by the manufacturer. The selective COX-1 inhibitor 5-(4-chlorophenyl)1-(4-methoxyphenyl)-3-trifluoromethylpyrazole (SC-560) and the selective COX-2 inhibitor 5-bromo-2-(4-fluorophenyl)-3-(4-methysulfonylphenyl) thiophene (DuP-697) were used as reference drugs. Cayman Chemicals supplied both the SC-560 and DuP-697. All measurements were carried out in duplicate and presented as the mean \pm the standard deviation. The in vitro COX-2 selectivity index SI-2 was calculated from the following formula [14]:

$$
S I-2=\frac{C O X-2 \text { inhibition }(\%)}{C O X-1 \text { inhibition }(\%)}
$$

\section{Molecular docking analysis}

\section{Ligand and protein preparation}

The 2D structures of 37-58 and 61-63 were sketched using the freeware ACD/ChemSketch version 12.01 (ACD/Labs Release, Canada). These were then converted into a standard structure-data file (SDF) format using the freely available open source toolbox, Open Babel 2.3.2. Energy minimisation was conducted using optimised potential for liquid simulations (OPLS3) force field [15] using the Lig Prep Module provided by Maestro (Schrödinger, LCC, New York, 2017).

The 3D structure of COX-1 (PDB code: 3N8Z, 2.9 $\AA$ ) and COX-2 (PDB code: 3NT1, $1.73 \AA$ ) were retrieved from Protein Data Bank (PDB) and processed using the Protein Preparation Wizard from Maestro. Protein preparation was executed for chain A only, while the other chain and non-proteinic molecules like heme, water and arachidonic acid were removed. Glide Receptor Grid Generation platform as a part of Maestro, was employed to create a rigid grid around the interacting residues of the active site from the prepared protein structure [16].

\section{Extra precision molecular docking and binding energy calculation}

To predict the binding pose of ligands $\mathbf{3 7 - 5 8}$ and $\mathbf{6 1 -}$ 63 with COX-1/COX-2, the extra precision (XP) docking procedure was applied using Glide workflow from Maestro [17]. The ligands were docked flexibly into the rigid docking box. Internal conformations like ring conformers, acyclic bond torsion and nitrogen inversion were generated during the docking process. For each ligand, one docking hit with the lowest glide docking score was generated and used to study the docking results. The docking poses generated from Glide XP docking were used as an input to calculate the binding energy. The binding free energy calculation was performed using Prime Molecular Mechanics/Generalised Born Surface Area MM-GBSA (Prime, Schrödinger, LCC, New York, 2017). All atoms of the receptor and ligands were optimised and minimised using VSGB 2.0 solvation model [18]. The binding energies were calculated by OPLS force field [15]. 


\section{Results}

Chemical synthesis

Synthesis of ethyl N-arylglycinate esters 6-10

Ethyl $N$-phenylglycinate ester 6: White shiny plates $(95 \%$ ethanol); $94.65 \%$ yield; $\mathrm{mp} 55-57{ }^{\circ} \mathrm{C}$; IR $\left(v_{\max }, \mathrm{cm}^{-1}\right)$ 3386 (N-H), 3050 (C-H ring), 2979-2865 (C-H aliphatic), 1732 ( $\mathrm{C}=\mathrm{O}), 1607-1518$ ( $\mathrm{C}=\mathrm{C}$ ring), 1229 (CO); ${ }^{1} \mathrm{H}$ NMR (700 MHz, DMSO- $\left.d_{6}\right) 7.11$ (dd, $J=7.53$, $7.37 \mathrm{~Hz}, 2 \mathrm{H}, \mathrm{C} 3-\mathrm{H}, \mathrm{C} 5-\mathrm{H}), 6.61$ (d, J = $7.38 \mathrm{~Hz}, 1 \mathrm{H}, \mathrm{C} 4-$ $\mathrm{H}), 6.59(\mathrm{~d}, J=8.02 \mathrm{~Hz}, 2 \mathrm{H}, \mathrm{C} 2-\mathrm{H}, \mathrm{C6}-\mathrm{H}), 5.95(\mathrm{t}, J=$ $6.33 \mathrm{~Hz}, 1 \mathrm{H}, \mathrm{NH}), 4.13\left(\mathrm{q}, J=7.11 \mathrm{~Hz}, 2 \mathrm{H}, \mathrm{OCH}_{2}\right.$ ), $3.90\left(\mathrm{~d}, J=6.67 \mathrm{~Hz}, 2 \mathrm{H}, \mathrm{NCH}_{2}\right), 1.21(\mathrm{t}, J=7.21 \mathrm{~Hz}, 3$ $\mathrm{H}, \mathrm{CH}_{3}$ ). Supplementary data (Spectrum S1-S2).

Ethyl $N$-(4-methylphenyl)glycinate ester 7: Colourless shiny plates (95\% ethanol); $84.04 \%$ yield; mp $39-43{ }^{\circ} \mathrm{C}$; IR $\left(v_{\max }, \mathrm{cm}^{-1}\right) 3380(\mathrm{~N}-\mathrm{H}), 3039$ (C-H ring), 2992-2864 (C-H aliphatic), $1728(\mathrm{C}=\mathrm{O}), 1621-1510$ ( $\mathrm{C}=\mathrm{C}$ ring), 1227 (C-O); ${ }^{1} \mathrm{H}$ NMR (700 MHz, DMSO-d $) 6.91$ (d, $J=$ $8.39 \mathrm{~Hz}, 2 \mathrm{H}, \mathrm{C} 3-\mathrm{H}, \mathrm{C} 5-\mathrm{H}), 6.49$ (d, $J=8.61 \mathrm{~Hz}, 2 \mathrm{H}$, C2-H, C6-H), 5.74 (t, $J=6.45 \mathrm{~Hz}, 1 \mathrm{H}, \mathrm{NH}$ ), 4.12 (q, $J=$ $\left.7.10 \mathrm{~Hz}, 2 \mathrm{H}, \mathrm{OCH}_{2}\right), 3.85\left(\mathrm{~d}, J=6.67 \mathrm{~Hz}, 2 \mathrm{H}, \mathrm{NCH}_{2}\right.$ ), 2.17 (s, $\left.3 \mathrm{H}, \mathrm{C} 4-\mathrm{CH}_{3}\right), 1.20\left(\mathrm{t}, J=7.21 \mathrm{~Hz}, 3 \mathrm{H}, \mathrm{CH}_{3}\right)$. Supplementary data (Spectrum S3-S4).

Ethyl $N$-(4-methoxyphenyl)glycinate ester 8: Off-white plates (95\% ethanol); $52.96 \%$ yield; mp $57-60{ }^{\circ} \mathrm{C}$; IR $\left(v_{\max }, \mathrm{cm}^{-1}\right) 3383(\mathrm{~N}-\mathrm{H}), 3039$ (C-H ring), 2989-2838 (C-H aliphatic), $1733(\mathrm{C}=\mathrm{O}), 1618-1517$ ( $\mathrm{C}=\mathrm{C}$ ring), 1213 (C-O); ${ }^{1} \mathrm{H}$ NMR (700 MHz, DMSO-d 6 ) 6.74 (d, $J=$ $8.82 \mathrm{~Hz}, 2 \mathrm{H}, \mathrm{C} 3-\mathrm{H}, \mathrm{C} 5-\mathrm{H}), 6.54(\mathrm{~d}, J=9.03 \mathrm{~Hz}, 2 \mathrm{H}$, C2-H, C6-H), 5.54 (t, $J=6.35 \mathrm{~Hz}, 1 \mathrm{H}, \mathrm{NH}), 4.12$ (q, $J=$ $\left.7.10 \mathrm{~Hz}, 2 \mathrm{H}, \mathrm{OCH}_{2}\right), 3.84\left(\mathrm{~d}, J=6.45 \mathrm{~Hz}, 2 \mathrm{H}, \mathrm{NCH}_{2}\right.$ ), 3.65 (s, $\left.3 \mathrm{H}, \mathrm{OCH}_{3}\right), 1.20$ (t, $J=7.42 \mathrm{~Hz}, 3 \mathrm{H}, \mathrm{CH}_{3}$ ). Supplementary data (Spectrum S5-S6).

Ethyl $N$-(4-chlorophenyl)glycinate ester 9: White shiny plates (95\% ethanol); $61.50 \%$ yield; mp $94-97{ }^{\circ} \mathrm{C}$; IR $\left(v_{\max }, \mathrm{cm}^{-1}\right) 3377(\mathrm{~N}-\mathrm{H}), 3052(\mathrm{C}-\mathrm{H}$ ring), 2987-2845 (C-H aliphatic), 1721 ( $\mathrm{C}=\mathrm{O}), 1608-1511$ ( $\mathrm{C}=\mathrm{C}$ ring), 1227 (C-O), 1021 (C-Cl); ${ }^{1} \mathrm{H}$ NMR (700 MHz, DMSOd6) $7.10(\mathrm{~d}, J=8.86 \mathrm{~Hz}, 2 \mathrm{H}, \mathrm{C} 3-\mathrm{H}, \mathrm{C} 5-\mathrm{H}), 6.57$ (d, $J=$ $8.86 \mathrm{~Hz}, 2 \mathrm{H}, \mathrm{C} 2-\mathrm{H}, \mathrm{C} 6-\mathrm{H}), 6.18$ (t, $J=6.30 \mathrm{~Hz}, 1 \mathrm{H}$, $\mathrm{NH}), 4.12$ (q, $\left.J=7.04 \mathrm{~Hz}, 2 \mathrm{H}, \mathrm{OCH}_{2}\right), 3.89$ (d, $J=6.47$ $\mathrm{Hz}, 2 \mathrm{H}, \mathrm{NCH}_{2}$ ), 1.19 (t, $J=7.15 \mathrm{~Hz}, 3 \mathrm{H}, \mathrm{CH}_{3}$ ). Supplementary data (Spectrum S7-S8).

Ethyl N-(4-fluorophenyl)glycinate ester 10: Yellowish transparent plates (95\% ethanol);79.11\% yield; $\mathrm{mp} 72-$ $75{ }^{\circ} \mathrm{C}$; IR $\left(v_{\max }, \mathrm{cm}^{-1}\right) 3383(\mathrm{~N}-\mathrm{H}), 3079-3046(\mathrm{C}-\mathrm{H}$ ring), 2993-2853 (C-H aliphatic), 1728 (C=O), 16181525 (C=C ring), 1220 (C-O), $1032(\mathrm{C}-\mathrm{F}) ;{ }^{1} \mathrm{H}$ NMR $\left(700 \mathrm{MHz}, \mathrm{DMSO}-d_{6}\right) 6.93(\mathrm{t}, J=8.99 \mathrm{~Hz}, 2 \mathrm{H}, \mathrm{C} 3-$ $\mathrm{H}, \mathrm{C} 5-\mathrm{H}$ ), 6.57 (dd, $J=9.03,4.51 \mathrm{~Hz}, 2 \mathrm{H}, \mathrm{C} 2-\mathrm{H}$, C6-H), $5.91(\mathrm{t}, J=6.35 \mathrm{~Hz}, 1 \mathrm{H}, \mathrm{NH}), 4.12(\mathrm{q}, J=$ $\left.7.03 \mathrm{~Hz}, 2 \mathrm{H}, \mathrm{OCH}_{2}\right), 3.88(\mathrm{~d}, J=6.45 \mathrm{~Hz}, 2 \mathrm{H}$, $\left.\mathrm{NCH}_{2}\right), 1.20\left(\mathrm{t}, J=7.31 \mathrm{~Hz}, 3 \mathrm{H}, \mathrm{CH}_{3}\right)$. Supplementary data (Spectrum S9-S10).

\section{Synthesis of methyl 4-aminobenzoate 12}

Methyl 4-aminobenzoate 12: Shiny white plates (95\% ethanol); 93.72\% yield; mp $111-113{ }^{\circ} \mathrm{C}$; IR $\left(v_{\max }, \mathrm{cm}^{-1}\right)$ 3411 (asymmetric N-H), 3336 (symmetric N-H), 3225 (overtone and combination), 3031 (C-H ring), 29882844 (aliphatic C-H), $1684(\mathrm{C}=\mathrm{O}), 1640-1602 \quad(\mathrm{C}=\mathrm{C}$ ring), $1292 \mathrm{~cm}^{-1}$ (C-C(=O)-O); ${ }^{1} \mathrm{H}$ NMR (700 MHz, DMSO-d $d_{6} 7.75$ (d, $\left.J=8.66 \mathrm{~Hz}, 2 \mathrm{H}, \mathrm{C} 2-\mathrm{H}, \mathrm{C} 6-\mathrm{H}\right), 6.82$ (d, $J=8.66 \mathrm{~Hz}, 2 \mathrm{H}, \mathrm{C} 3-\mathrm{H}, \mathrm{C} 5-\mathrm{H}$ ), 6.30 (br. s., $2 \mathrm{H}, \mathrm{NH}$ ), $3.76\left(\mathrm{~s}, 3 \mathrm{H}, \mathrm{OCH}_{3}\right)$. Supplementary data (Spectrum S11-S12).

\section{Synthesis of $\mathrm{N}$-arylglycine 13-17}

$N$-Phenylglycine 13: White solid; $84.74 \%$ yield; mp $117-$ $119{ }^{\circ} \mathrm{C}$; IR $\left(v_{\max }, \mathrm{cm}^{-1}\right) 3445(\mathrm{~N}-\mathrm{H}), 3129-1887(\mathrm{O}-\mathrm{H})$, 3025 (C-H ring), 2948-2803 (C-H aliphatic), 1565 (asymmetric $\mathrm{COO}^{-}$), 1494 ( $\mathrm{C}=\mathrm{C}$ ring), 1384 (symmetric $\mathrm{COO}^{-}$), 764, 695 (out-of-plane $\mathrm{C}-\mathrm{H}$ ring bending); ${ }^{1} \mathrm{H}$ NMR (700 MHz, DMSO- $\left.d_{6}\right) 7.07(\mathrm{t}, J=7.77 \mathrm{~Hz}, 2 \mathrm{H}$, C3-H, C5-H), 6.56 (d, J = 7.53 Hz, $1 \mathrm{H}, \mathrm{C} 4-\mathrm{H}), 6.54$ (d, J $=7.85 \mathrm{~Hz}, 2 \mathrm{H}, \mathrm{C} 2-\mathrm{H}, \mathrm{C} 6-\mathrm{H}), 3.78$ (s, $\left.2 \mathrm{H}, \mathrm{CH}_{2} \mathrm{CO}\right)$. Supplementary data (Spectrum S13-S14).

$N$-(4-Methylphenyl)glycine 14: White solid; $54.06 \%$ yield; mp 105-108 ${ }^{\circ} \mathrm{C}$; IR $\left(v_{\max }, \mathrm{cm}^{-1}\right) 3433(\mathrm{~N}-\mathrm{H})$, 3170-1837 (O-H), 3043 (C-H ring), 2971-2793 (C-H aliphatic), 1595 (asymmetric $\mathrm{COO}^{-}$), 1512 ( $\mathrm{C}=\mathrm{C}$ ring), 1398 (symmetric $\mathrm{COO}^{-}$), 821 (out-of-plane $\mathrm{C}-\mathrm{H}$ ring bending); ${ }^{1} \mathrm{H}$ NMR (700 MHz, DMSO- $\left.d_{6}\right) 6.88$ (d, $J=$ $8.39 \mathrm{~Hz}, 2 \mathrm{H}, \mathrm{C} 3-\mathrm{H}, \mathrm{C} 5-\mathrm{H}), 6.45$ (d, $J=8.39 \mathrm{~Hz}, 2 \mathrm{H}$, C2-H, C6-H), 3.74 (s, $2 \mathrm{H}, \mathrm{CH}_{2} \mathrm{CO}$ ), 2.14 (s, $3 \mathrm{H}, \mathrm{CH}_{3}$ ). Supplementary data (Spectrum S15-S16).

$N$-(4-Methoxyphenyl)glycine 15: White solid; $68.91 \%$ yield; mp 148-149 ${ }^{\circ} \mathrm{C}$; IR $\left(v_{\max }, \mathrm{cm}^{-1}\right) 3428(\mathrm{~N}-\mathrm{H})$, 3190-1823 (O-H), 3061, 3025 (C-H ring), 2998-2798 (C-H aliphatic), 1608 (asymmetric $\mathrm{COO}^{-}$), $1515(\mathrm{C}=\mathrm{C}$ ring), 1389 (symmetric $\mathrm{COO}^{-}$), 839 (out-of-plane $\mathrm{C}-\mathrm{H}$ ring bending); ${ }^{1} \mathrm{H}$ NMR (700 MHz, DMSO- $\left.d_{6}\right) 6.71(\mathrm{~d}, J$ $=8.82 \mathrm{~Hz}, 2 \mathrm{H}, \mathrm{C} 3-\mathrm{H}, \mathrm{C} 5-\mathrm{H}), 6.51(\mathrm{~d}, J=8.82 \mathrm{~Hz}, 2 \mathrm{H}$, C2-H, C6-H), 3.72 (s, 2 H, $\mathrm{CH}_{2} \mathrm{CO}$ ), 3.63 (s, $3 \mathrm{H}$, $\left.\mathrm{OCH}_{3}\right)$. Supplementary data (Spectrum S17-S18).

N-(4-Chlorophenyl)glycine 16: White solid; $90.27 \%$ yield; mp $138{ }^{\circ} \mathrm{C}$; IR $\left(v_{\max }, \mathrm{cm}^{-1}\right) 3416(\mathrm{~N}-\mathrm{H}), 3263-$ 1919 (O-H), 3078-3045 (C-H ring), 2991-2824 (C-H aliphatic), $1709(\mathrm{C}=\mathrm{O}), 1601,1502$ ( $\mathrm{C}=\mathrm{C}$ ring), 812 (outof-plane $\mathrm{C}-\mathrm{H}$ ring bending), $1084(\mathrm{C}-\mathrm{Cl}) ;{ }^{1} \mathrm{H}$ NMR (700 MHz, DMSO- $\left.d_{6}\right) 7.09(\mathrm{~d}, J=8.86 \mathrm{~Hz}, 2 \mathrm{H}, \mathrm{C} 3-\mathrm{H}, \mathrm{C} 5-$ $\mathrm{H}), 6.56$ (d, $J=8.86 \mathrm{~Hz}, 2 \mathrm{H}, \mathrm{C} 2-\mathrm{H}, \mathrm{C} 6-\mathrm{H}), 3.79$ (s, $2 \mathrm{H}$, $\mathrm{CH}_{2} \mathrm{CO}$ ). Supplementary data (Spectrum S19-S20).

$N$-(4-Fluorophenyl)glycine 17: White solid; $64.74 \%$ yield; mp 131-134 ${ }^{\circ} \mathrm{C}$; IR $\left(v_{\max }, \mathrm{cm}^{-1}\right) 3446(\mathrm{~N}-\mathrm{H})$, 3128-1981 (O-H), 3019 (C-H ring), 2911-2816 (C-H aliphatic), 1574 (asymmetric $\mathrm{COO}^{-}$), 1515 ( $\mathrm{C}=\mathrm{C}$ ring), 1387 (symmetric $\mathrm{COO}^{-}$), 841 (out-of-plane $\mathrm{C}-\mathrm{H}$ ring bending); ${ }^{1} \mathrm{H}$ NMR (700 MHz, DMSO- $\left.d_{6}\right) 6.91$ ( $\mathrm{t}, J=$ 
$8.61 \mathrm{~Hz}, 2 \mathrm{H}, \mathrm{C} 3-\mathrm{H}, \mathrm{C} 5-\mathrm{H}), 6.54(\mathrm{dd}, J=9.03,4.51 \mathrm{~Hz}, 2$ $\mathrm{H}, \mathrm{C} 2-\mathrm{H}, \mathrm{C} 6-\mathrm{H}), 3.76$ (s, $2 \mathrm{H}, \mathrm{CH}_{2} \mathrm{CO}$ ). Supplementary data (Spectrum S21-S22).

\section{Synthesis of nitro-containing $\mathrm{N}$-arylglycine 22-25}

$N$-(3-Nitrophenyl)glycine 22: Deep yellow plates (95\% ethanol); $58.79 \%$ yield; $\mathrm{mp} 156-158{ }^{\circ} \mathrm{C}$; IR $\left(v_{\max }, \mathrm{cm}^{-1}\right)$ 3401 (N-H), 3192-2190 (O-H), 3091 (C-H ring), 29922929 (C-H aliphatic), 1712 (C=O), 1541 (asymmetric NO), 1619-1417 ( $\mathrm{C}=\mathrm{C}$ ring), 1351 (symmetric $\mathrm{N}-\mathrm{O}$ ), 855, 808, 733 (out-of-plane C-H ring bending); ${ }^{1} \mathrm{H}$ NMR (700 MHz, DMSO-d $_{6} 12.70$ (br.s., $1 \mathrm{H}, \mathrm{COOH}$ ), 7.38 (dd, $J=$ 7.85, $1.61 \mathrm{~Hz}, 1 \mathrm{H}, \mathrm{C} 4-\mathrm{H}), 7.35$ (s, 1H, C2-H); 7.34 (t, $J=$ $6.65 \mathrm{~Hz}, 1 \mathrm{H}, \mathrm{C} 5-\mathrm{H}), 7.00$ (dd, $J=8.06,1.83 \mathrm{~Hz}, 1 \mathrm{H}, \mathrm{C6}-$ $\mathrm{H}), 6.68$ (br.s., $1 \mathrm{H}, \mathrm{NH}$ ), 3.92 (s, $2 \mathrm{H}, \mathrm{CH}_{2} \mathrm{CO}$ ). Supplementary data (Spectrum S23-S24).

$N$-(4-Nitrophenyl)glycine 23: Yellow plates (95\% ethanol); $50.58 \%$ yield; $\mathrm{mp} 221-223{ }^{\circ} \mathrm{C}$; IR $\left(v_{\max }, \mathrm{cm}^{-1}\right) 3367$ (N-H), 3282-2500 (O-H), 3103 (C-H ring), 2933-2891 (C-H aliphatic), $1735(\mathrm{C}=\mathrm{O}), 1537$ (asymmetric N-O), 1603-1471 ( $\mathrm{C}=\mathrm{C}$ ring), 1305 (symmetric $\mathrm{N}-\mathrm{O}$ ), 823 (out-of-plane $\mathrm{C}-\mathrm{H}$ ring bending); ${ }^{1} \mathrm{H}$ NMR (700 $\mathrm{MHz}$, DMSO- $d_{6}$ ) 12.82 (br.s., $\left.1 \mathrm{H}, \mathrm{COOH}\right), 8.01$ (d, $J=9.25$ $\mathrm{Hz}, 2 \mathrm{H}, \mathrm{C} 3-\mathrm{H}, \mathrm{C} 5-\mathrm{H}), 7.47$ (t, $J=6.02 \mathrm{~Hz}, 1 \mathrm{H}, \mathrm{NH}), 6.67$ (d, $J=9.04 \mathrm{~Hz}, 2 \mathrm{H}, \mathrm{C} 2-\mathrm{H}, \mathrm{C} 6-\mathrm{H}), 3.98$ (d, $J=6.02 \mathrm{~Hz}$, $2 \mathrm{H}, \mathrm{CH}_{2} \mathrm{CO}$ ). Supplementary data (Spectrum S25-S26).

$N$-(4-Methyl-3-nitrophenyl)glycine 24: Yellow plates (95\% ethanol); $82.81 \%$ yield; $\mathrm{mp} 141-143{ }^{\circ} \mathrm{C}$; IR ( $v_{\max }$, $\left.\mathrm{cm}^{-1}\right) 3381$ (N-H), 3272-1983 (O-H), 3086, 3039 (C-H ring), 2983-2872 (C-H aliphatic), 1717 ( $\mathrm{C}=\mathrm{O}), 1541$ (asymmetric N-O), 1626-1417 ( $\mathrm{C}=\mathrm{C}$ ring), 1336 (symmetric N-O), 891, 824 (out-of-plane $\mathrm{C}-\mathrm{H}$ ring bending); ${ }^{1} \mathrm{H}$ NMR (700 MHz, DMSO-d 6 ) 12.65 (br.s., $1 \mathrm{H}$, $\mathrm{COOH}), 7.17(\mathrm{~d}, J=8.17 \mathrm{~Hz}, 1 \mathrm{H}, \mathrm{C} 5-\mathrm{H}), 7.12(\mathrm{~d}, J=$ $2.58 \mathrm{~Hz}, 1 \mathrm{H}, \mathrm{C} 2-\mathrm{H}), 6.85$ (dd, $J=8.28,2.61 \mathrm{~Hz}, 1 \mathrm{H}, \mathrm{C6}-$ $\mathrm{H}), 6.39$ (br.s., $1 \mathrm{H}, \mathrm{NH}$ ), 3.87 (s, $2 \mathrm{H}, \mathrm{CH}_{2} \mathrm{CO}$ ), 2.33 (s, $3 \mathrm{H}, \mathrm{C} 4-\mathrm{CH}_{3}$ ). Supplementary data (Spectrum S27-S28).

N-(4-Chloro-3-nitrophenyl)glycine 25: Yellow plates (isopropanol);71.03\% yield; $\mathrm{mp}$ 174-177 ${ }^{\circ} \mathrm{C}$; IR ( $v_{\max }$, $\mathrm{cm}^{-1}$ ) $3386(\mathrm{~N}-\mathrm{H}), 3282-1947(\mathrm{O}-\mathrm{H}), 3085,3057$ (C-H ring), 2971-2848 ( $\mathrm{C}-\mathrm{H}$ aliphatic), $1721(\mathrm{C}=\mathrm{O}), 1537$ (asymmetric N-O), 1617-1442 ( $\mathrm{C}=\mathrm{C}$ ring), 1338 (symmetric N-O), 891, 822 (out-of-plane $\mathrm{C}-\mathrm{H}$ ring bending); ${ }^{1} \mathrm{H}$ NMR (700 MHz, DMSO-d $) 12.73$ (br.s., $1 \mathrm{H}$, $\mathrm{COOH}), 7.38(\mathrm{~d}, J=9.94 \mathrm{~Hz}, 1 \mathrm{H}, \mathrm{C} 5-\mathrm{H}), 7.15(\mathrm{~d}, J=$ $2.80 \mathrm{~Hz}, 1 \mathrm{H}, \mathrm{C} 2-\mathrm{H}), 6.88$ (dd, $J=9.03,2.80 \mathrm{~Hz}, 1 \mathrm{H}, \mathrm{C6}-$ $\mathrm{H}), 6.74(\mathrm{t}, J=5.92 \mathrm{~Hz}, 1 \mathrm{H}, \mathrm{NH}), 3.90(\mathrm{~d}, J=5.81 \mathrm{~Hz}$, $2 \mathrm{H}, \mathrm{CH}_{2} \mathrm{CO}$ ). Supplementary data (Spectrum S29-S30).

\section{Synthesis of N-[4-(methoxycarbonyl)phenyl]glycine 26}

$N$-[4-(methoxycarbonyl)phenyl]glycine 26: White solid; $53.12 \%$ yield; $\mathrm{mp} 171-174{ }^{\circ} \mathrm{C}$; IR $\left(v_{\max }, \mathrm{cm}^{-1}\right)$ 34332480 (O-H), 3335 (N-H), 3094-3004 (C-H ring), 29972883 (C-H aliphatic), 1735 (CO acid), 1673 (CO ester),
1603-1437 ( $\mathrm{C}=\mathrm{C}$ ring), 1317 ( $\mathrm{C}(=\mathrm{O})-\mathrm{O}$ acid), 1292, 1178 (C-C(=O)-O ester), 846 (out-of-plane $\mathrm{C}-\mathrm{H}$ bending ring); ${ }^{1} \mathrm{H}$ NMR (700 MHz, DMSO- $\left.d_{6}\right) 7.71(\mathrm{~d}, J=8.82 \mathrm{~Hz}, 2$ H, C3-H, C5-H), 6.75 (br. s., $1 \mathrm{H}, \mathrm{N}-\mathrm{H}$ ), 6.61 (d, J = 8.82 $\mathrm{Hz}, 2 \mathrm{H}, \mathrm{C} 2-\mathrm{H}, \mathrm{C} 6-\mathrm{H}$ ), 3.89 (s, $2 \mathrm{H}, \mathrm{CH}_{2} \mathrm{CO}$ ), 3.74 (s, 3 $\mathrm{H}, \mathrm{OCH}_{3}$ ). Supplementary data (Spectrum S31-S32).

\section{Synthesis of N-nitroso-N-arylglycine 27-36}

$N$-Nitroso- $N$-phenylglycine 27: Pale yellow solid; $69.06 \%$ yield; mp 99-101 ${ }^{\circ} \mathrm{C}$; IR $\left(v_{\max }, \mathrm{cm}^{-1}\right)$ 3427-2255 (O-H), 1731 ( $\mathrm{C}=\mathrm{O}$ acid), 1436 (N=O), 1295 (C-O acid). Supplementary data (Spectrum S33).

$N$-Nitroso- $N$-(4-methylphenyl)glycine 28: Off-white solid; $90.05 \%$ yield; $\mathrm{mp} 101-102{ }^{\circ} \mathrm{C}$; IR $\left(v_{\max }, \mathrm{cm}^{-1}\right)$ 3337-2343 (O-H), 1728 (C=O acid), 1440 ( N=O), 1292 (C-O acid). Supplementary data (Spectrum S34).

$N$-Nitroso- $N$-(4-methoxyphenyl)glycine 29: Off-white solid; $94.19 \%$ yield; $\mathrm{mp} 113-114{ }^{\circ} \mathrm{C}$; IR $\left(v_{\max }, \mathrm{cm}^{-1}\right)$ 3290-2406 (O-H), 1720 (C=O acid), 1448 (N=O), 1302 (C-O acid). Supplementary data (Spectrum S35).

$N$-Nitroso- $N$-(4-chlorophenyl)glycine 30: Pale yellow solid; $91.50 \%$ yield; $\mathrm{mp} 112-115{ }^{\circ} \mathrm{C}$; IR $\left(v_{\max }, \mathrm{cm}^{-1}\right)$ 3417-2366 (O-H), 1730 (C=O acid), 1467 ( $\mathrm{N}=\mathrm{O}), 1306$ (C-O acid). Supplementary data (Spectrum S36).

$N$-Nitroso- $N$-(4-Fluorophenyl)glycine 31 :Yellowish solid; $35.80 \%$ yield; $\mathrm{mp} 97-99{ }^{\circ} \mathrm{C}$; IR $\left(v_{\max }, \mathrm{cm}^{-1}\right) 3419-$ 2471 (O-H), 1726 (C=O acid), 1446 (N=O), 1302 (C-O acid). Supplementary data (Spectrum S37).

$N$-Nitroso- $N$-(3-nitrophenyl)glycine 32: Pale yellow solid; $80.81 \%$ yield; $\mathrm{mp} 156-157{ }^{\circ} \mathrm{C}$; IR $\left(v_{\max }, \mathrm{cm}^{-1}\right)$ 3447-2405 (O-H), 1722 ( $\mathrm{C}=\mathrm{O}$ acid), 1440 (N=O), 1280 (C-O acid). Supplementary data (Spectrum S38).

$N$-Nitroso- $N$-(4-nitrophenyl)glycine 33: Pale yellow solid; $91.25 \%$ yield; $\mathrm{mp} 138{ }^{\circ} \mathrm{C}$; IR $\left(v_{\max }, \mathrm{cm}^{-1}\right)$ 3391$2409(\mathrm{O}-\mathrm{H}), 1731(\mathrm{C}=\mathrm{O}$ acid$), 1486(\mathrm{~N}=\mathrm{O}), 1305$ (C-O acid). Supplementary data (Spectrum S39).

$N$-Nitroso- $N$-(4-methyl-3-nitrophenyl)glycine 34:Yellow solid;79.88\% yield; mp $127-128{ }^{\circ} \mathrm{C}$; IR $\left(v_{\max }, \mathrm{cm}^{-1}\right)$ 3429-2527 (O-H), 1730 (C=O acid), $1472(\mathrm{~N}=\mathrm{O}), 1282$ (C-O acid). Supplementary data (Spectrum S40).

$N$-Nitroso- $N$-(4-chloro-3-nitrophenyl)glycine 35:Yellow solid;79.88\% yield; mp 127-128 ${ }^{\circ} \mathrm{C}$; IR $\left(v_{\max }, \mathrm{cm}^{-1}\right)$ 3348-2702 (O-H), 1730 (C=O acid), 1469 (N=O), 1298 (C-O acid). Supplementary data (Spectrum S41).

$N$-Nitroso- $N$-[4-(methoxycarbonyl)phenyl]glycine 36 : Yellow solid; $91.12 \%$ yield; mp $125{ }^{\circ} \mathrm{C}$; IR $\left(v_{\max }, \mathrm{cm}^{-1}\right)$ 3409-2433 (O-H), 1714 ( $\mathrm{C}=\mathrm{O}$ acid), 1432 (N=O), 1308 (C-O acid). Supplementary data (Spectrum S42).

\section{Synthesis of 3-arylsydnones 37-46}

3-Phenylsydnone 37: Pale yellow needles (95\% ethanol); 89.21\% yield; mp $134-135{ }^{\circ} \mathrm{C}$; IR $\left(v_{\max }, \mathrm{cm}^{-1}\right) 3128(\mathrm{C}-\mathrm{H}$ sydnone), 3077, 3064 ( $\mathrm{C}-\mathrm{H}$ ring), 1760 ( $\mathrm{C}=\mathrm{O}$ sydnone), 1497-1440 ( $\mathrm{C}=\mathrm{C}$ ring), 727, 682 (out-of-plane $\mathrm{C}-\mathrm{H}$ ring 
bending); ${ }^{1} \mathrm{H}$ NMR $\left(700 \mathrm{MHz}, \mathrm{CDCl}_{3}\right) 7.74(\mathrm{~d}, J=7.83$ $\mathrm{Hz}, 2 \mathrm{H}, \mathrm{C} 7-\mathrm{H}, \mathrm{C} 11-\mathrm{H}), 7.33$ (t, $J=7.33 \mathrm{~Hz}, 1 \mathrm{H}, \mathrm{C} 9-\mathrm{H})$, 7.65 (t, $J=7.67 \mathrm{~Hz}, 2 \mathrm{H}, \mathrm{C} 8-\mathrm{H}, \mathrm{C} 10-\mathrm{H}), 6.78$ (s, $1 \mathrm{H}, \mathrm{C} 4-$ $\mathrm{H}) ;{ }^{13} \mathrm{C}$ NMR (176 MHz, CDCl $\left.l_{3}\right) 169.03$ (C5), 134.82 (C6), 132.52 (C9), 130.32 (C7, C11), 121.33 (C8, C10), 93.76 (C4). Supplementary data (Spectrum S43-S45).

3-(4-Methylphenyl)sydnone 38: White needles (95\% ethanol); $92.17 \%$ yield; $\mathrm{mp} 141-143{ }^{\circ} \mathrm{C}$; IR $\left(v_{\max }, \mathrm{cm}^{-1}\right)$ 3139 (C-H sydnone), 3080, 3054 (C-H benzene), 1754 ( $\mathrm{C}=\mathrm{O}$ sydnone), 1509, 1447 ( $\mathrm{C}=\mathrm{C}$ benzene), 817 (out-ofplane $\mathrm{C}-\mathrm{H}$ benzene bending); ${ }^{1} \mathrm{H}$ NMR $(700 \mathrm{MHz}$, $\left.\mathrm{CDCl}_{3}\right) 7.61(\mathrm{~d}, J=8.51 \mathrm{~Hz}, 2 \mathrm{H}, \mathrm{C} 7-\mathrm{H}, \mathrm{C} 11-\mathrm{H}), 7.41(\mathrm{~d}$, $J=8.18 \mathrm{~Hz}, 2 \mathrm{H}, \mathrm{C} 8-\mathrm{H}, \mathrm{C} 10-\mathrm{H}), 6.74(\mathrm{~s}, 1 \mathrm{H}, \mathrm{C} 4-\mathrm{H})$, 2.48 (s, $3 \mathrm{H}, \mathrm{C} 12) ;{ }^{13} \mathrm{C}$ NMR $\left(176 \mathrm{MHz}, \mathrm{CDCl}_{3}\right) 169.12$ (C5), 143.31 (C9), 132.47 (C6), 130.78 (C7, C11), 121.03 (C8, C10), 93.56 (C4), 21.39 (C12). Supplementary data (Spectrum S46-S48).

3-(4-Methoxyphenyl)sydnone 39: White needles (95\% ethanol); $86.08 \%$ yield; $\mathrm{mp} 124-126{ }^{\circ} \mathrm{C}$; IR $\left(v_{\max }, \mathrm{cm}^{-1}\right)$ 3150 (C-H sydnone), 3073 (C-H benzene), $1742(\mathrm{C}=\mathrm{O}$ sydnone), 1603-1457 ( $\mathrm{C}=\mathrm{C}$ benzene), 1259, 1008 (C-O$\mathrm{C}$ alkyl aryl ether), 834 (out-of-plane $\mathrm{C}-\mathrm{H}$ benzene bending); ${ }^{1} \mathrm{H}$ NMR $\left(700 \mathrm{MHz}, \mathrm{CDCl}_{3}\right) 7.65(\mathrm{~d}, J=8.86 \mathrm{~Hz}, 2$ $\mathrm{H}, \mathrm{C} 7-\mathrm{H}, \mathrm{C} 11-\mathrm{H}), 7.08$ (d, J = 9.20 Hz, $2 \mathrm{H}, \mathrm{C} 8-\mathrm{H}, \mathrm{C} 10-$ $\mathrm{H}), 6.72(\mathrm{~s}, 1 \mathrm{H}, \mathrm{C} 4-\mathrm{H}), 3.90(\mathrm{~s}, 3 \mathrm{H}, \mathrm{C} 12) ;{ }^{13} \mathrm{C} \mathrm{NMR}$ $\left(176 \mathrm{MHz}, C D C l_{3}\right) 169.16$ (C5), 162.51 (C9), 127.72 (C6), 122.70 (C7, C11), 115.26 (C8, C10), 93.49 (C4), 55.88 (C12). Supplementary data (Spectrum S49-S51).

3-(4-Chlorophenyl)sydnone 40: Pale yellow needles (95\% ethanol);70.63\% yield; mp $111-113{ }^{\circ} \mathrm{C}$; IR ( $v_{\max }$, $\left.\mathrm{cm}^{-1}\right) 3133$ (C-H sydnone), 3073, 3051 (C-H benzene), 1746 ( $\mathrm{C}=\mathrm{O}$ sydnone), $1493-1421(\mathrm{C}=\mathrm{C}$ benzene $), 1097$ (C-Cl), 832 (out-of-plane $\mathrm{C}-\mathrm{H}$ benzene bending); ${ }^{1} \mathrm{H}$ NMR $\left(700 \mathrm{MHz}, \mathrm{CDCl}_{3}\right) 7.73(\mathrm{~d}, J=9.19 \mathrm{~Hz}, 2 \mathrm{H}, \mathrm{C} 7-$ $\mathrm{H}, \mathrm{C} 11-\mathrm{H}), 7.62(\mathrm{~d}, J=8.86 \mathrm{~Hz}, 2 \mathrm{H}, \mathrm{C} 8-\mathrm{H}, \mathrm{C} 10-\mathrm{H})$, 6.81 (s, $1 \mathrm{H}, \mathrm{C} 4-\mathrm{H}) ;{ }^{13} \mathrm{C}$ NMR $\left(176 \mathrm{MHz}, \mathrm{CDCl}_{3}\right) 168.84$ (C5), 138.78 (C9), 133.19 (C6), 130.60 (C7, C11), 122.60 (C8, C10), 93.75 (C4). Supplementary data (Spectrum S52-S54).

3-(4-Fluorophenyl)sydnone 41: Pale yellow needles (95\% ethanol);75.88\% yield; $\mathrm{mp} 154-156{ }^{\circ} \mathrm{C}$; IR ( $v_{\max }$, $\mathrm{cm}^{-1}$ ) $3112 \mathrm{~cm}^{-1}$ (C-H sydnone), $3081 \mathrm{~cm}^{-1}$ (C-H benzene), $1733 \mathrm{~cm}^{-1}$ (C=O sydnone), 1598, 1510, 1455 (C= $\mathrm{C}$ benzene), $1021 \mathrm{~cm}^{-1}$ (C-F), 839 (out-of-plane $\mathrm{C}-\mathrm{H}$ benzene bending); ${ }^{1} \mathrm{H}$ NMR ${ }^{1} \mathrm{H}$ NMR $\left(700 \mathrm{MHz}, C D C l_{3}\right.$ ) 7.78 (dd, $J=9.19,4.43 \mathrm{~Hz}, 2 \mathrm{H}, \mathrm{C} 7-\mathrm{H}, \mathrm{C} 11-\mathrm{H}), 7.34$ (t, $J$ $=8.34 \mathrm{~Hz}, 2 \mathrm{H}, \mathrm{C} 8-\mathrm{H}, \mathrm{C} 10-\mathrm{H}), 6.78(\mathrm{~s}, 1 \mathrm{H}, \mathrm{C} 4-\mathrm{H}) ;{ }^{13} \mathrm{C}$ NMR $\left(176 \mathrm{MHz}, \mathrm{CDCl}_{3}\right) 168.92(\mathrm{C} 5), 164.56$ (d, $J=$ $254.31 \mathrm{~Hz}, \mathrm{C} 9), 130.93$ (C6), 123.62 (d, $J=8.90 \mathrm{~Hz}, \mathrm{C} 7$, C11), 117.580 (d, $J=24.16$, C8, C10), 93.96 (C4). Supplementary data (Spectrum S55-S57).

3-(3-Nitroyphenyl)sydnone 42: Tan plates (95\% ethanol); $80.41 \%$ yield; $\mathrm{mp} 140-142{ }^{\circ} \mathrm{C}$; IR $\left(v_{\max }, \mathrm{cm}^{-1}\right) 3155$ (C-H sydnone), 3116, 3062 (C-H benzene), 1755 (C=O sydnone), 1532 (asymmetric $\mathrm{N}=\mathrm{O}), 1492,1442(\mathrm{C}=\mathrm{C}$ benzene), 1359 (symmetric $\mathrm{N}=\mathrm{O}$ ), 817, 724, 690 (out-ofplane $\mathrm{C}-\mathrm{H}$ benzene ring), ${ }^{1} \mathrm{H}$ NMR $(700 \mathrm{MHz}$, DMSO$\left.d_{6}\right) 8.77(\mathrm{t}, J=2.05 \mathrm{~Hz}, 1 \mathrm{H}, \mathrm{C} 7-\mathrm{H}), 8.57$ (dd, $J=8.28$, $1.83 \mathrm{~Hz}, 1 \mathrm{H}, \mathrm{C} 9-\mathrm{H}), 8.41(\mathrm{dd}, J=8.18,1.73 \mathrm{~Hz}, 1 \mathrm{H}$, C11-H), $8.02(\mathrm{t}, J=8.18 \mathrm{~Hz}, 1 \mathrm{H}, \mathrm{C} 10-\mathrm{H}), 8.00(\mathrm{~s}, 1 \mathrm{H}$, C4-H); ${ }^{13} \mathrm{C}$ NMR (176 MHz, DMSO- $\left.d_{6}\right) 168.83$ (C5), 148.78 (C8), 135.53 (C6), 132.31 (C11), 128.43 (C10), 127.36 (C7), 117.69 (C9), 96.36 (C4). Supplementary data (Spectrum S58-S60).

3-(4-Nitroyphenyl)sydnone 43: Yellow needles (95\% ethanol); $90.01 \%$ yield; $\mathrm{mp} 190-192{ }^{\circ} \mathrm{C}$; IR $\left(v_{\max }, \mathrm{cm}^{-1}\right)$ 3118 (C-H sydnone), 1755 ( $\mathrm{C}=\mathrm{O}$ sydnone), 1535 (asymmetric $\mathrm{N}=\mathrm{O}), 1459$ ( $\mathrm{C}=\mathrm{C}$ benzene), 1346 (symmetric $\mathrm{N}=$ $\mathrm{O}), 849$ (out-of-plane $\mathrm{C}-\mathrm{H}$ benzene ring); ${ }^{1} \mathrm{H}$ NMR (700 $\left.\mathrm{MHz}, \mathrm{DMSO}-d_{6}\right) 8.54(\mathrm{~d}, J=8.86 \mathrm{~Hz}, 2 \mathrm{H}, \mathrm{C} 8-\mathrm{H}, \mathrm{C} 10-$ $\mathrm{H}), 8.24(\mathrm{~d}, J=9.20 \mathrm{~Hz}, 2 \mathrm{H}, \mathrm{C} 7-\mathrm{H}, \mathrm{C} 11-\mathrm{H}), 7.97(\mathrm{~s}, 1$ $\mathrm{H}, \mathrm{C} 4-\mathrm{H}) ;{ }^{13} \mathrm{C}$ NMR (176 MHz, DMSO- $\left.d_{6}\right) 168.83$ (C5), 149.86 (C9), 139.00 (C6), 126.00 (C7, C11), 123.69 (C8, C10), 96.27 (C4). Supplementary data (Spectrum S61S63).

3-(4-Methyl-3-nitroyphenyl)sydnone 44: Yellow plates (95\% ethanol); $65.79 \%$ yield; $\mathrm{mp} 120-121{ }^{\circ} \mathrm{C}$; IR ( $v_{\max }$, $\mathrm{cm}^{-1}$ ) 3122 (C-H sydnone), 3100, 3065 (C-H benzene), 1759 ( $\mathrm{C}=\mathrm{O}$ sydnone), 1537 (asymmetric $\mathrm{N}=\mathrm{O}$ ), 1504, 1491 ( $\mathrm{C}=\mathrm{C}$ benzene), 1363 (symmetric $\mathrm{N}=\mathrm{O}$ ), 896, 832 (out-of-plane $\mathrm{C}-\mathrm{H}$ benzene ring); ${ }^{1} \mathrm{H}$ NMR $(700 \mathrm{MHz}$, DMSO- $\left.d_{6}\right) 8.59(\mathrm{~d}, J=2.36 \mathrm{~Hz}, 1 \mathrm{H}, \mathrm{C} 7-\mathrm{H}),, 8.21(\mathrm{dd}, J$ = 8.28, $2.27 \mathrm{~Hz}, 1 \mathrm{H}, \mathrm{C} 11-\mathrm{H}), 7.91(\mathrm{~s}, 1 \mathrm{H}, \mathrm{C} 4-\mathrm{H}), 7.86$ $(\mathrm{d}, J=8.38,1 \mathrm{H}, \mathrm{C} 10-\mathrm{H}), 2.62(\mathrm{~s}, 3 \mathrm{H}, \mathrm{C} 12-\mathrm{H}) ;{ }^{13} \mathrm{C}$ NMR (176 MHz, DMSO-d $\left.d_{6}\right) 168.83$ (C5), 149.56 (C8), 137.64 (C6), 135.05 (C11), 133.32 (C9), 126.23 (C10), 118.39 (C7), 95.97 (C4), 19.85 (C12). Supplementary data (Spectrum S64-S66).

3-(4-Chloro-3-nitrophenyl)sydnone 45: Pale yellow plates (95\% ethanol); $90.61 \%$ yield; $\mathrm{mp} 176{ }^{\circ} \mathrm{C}$; IR ( $v_{\max }$, $\mathrm{cm}^{-1}$ ) 3120 (C-H sydnone), 3081, 3036 (C-H benzene), 1745 ( $\mathrm{C}=\mathrm{O}$ sydnone), 1543 (asymmetric $\mathrm{N}=\mathrm{O}), 1487$, 1453 ( $\mathrm{C}=\mathrm{C}$ benzene), 1355 (symmetric $\mathrm{N}=\mathrm{O}$ ), 892, 845 (out-of-plane $\mathrm{C}-\mathrm{H}$ benzene ring); ${ }^{1} \mathrm{H}$ NMR $(700 \mathrm{MHz}$, DMSO- $\left.d_{6}\right) 8.77(\mathrm{~d}, J=2.58 \mathrm{~Hz}, \mathrm{C} 7-\mathrm{H}), 8.30(\mathrm{dd}, J=$ 8.82, $2.58 \mathrm{~Hz}, \mathrm{C} 11-\mathrm{H}), 8.17$ (d, $J=8.82 \mathrm{~Hz}, \mathrm{C} 10-\mathrm{H}), 7.93$ (s, $1 \mathrm{H}, \mathrm{C} 4-\mathrm{H}) ;{ }^{13} \mathrm{C}$ NMR (176 MHz, DMSO-d $\left.{ }_{6}\right) 168.70$ (C5), 148.32 (C8), 134.01 (C6), 133.97 (C11), 129.22 (C9), 127.07 (C10), 119.82 (C7), 96.26 (C4). Supplementary data (Spectrum S67-S69).

3-[4-(Methoxycarbonyl)phenyl]sydnone 46: White creamy needles (95\% ethanol);87.65\% yield; $\mathrm{mp} 180{ }^{\circ} \mathrm{C}$; IR $\left(v_{\max }, \mathrm{cm}^{-1}\right) 3130$ (C-H sydnone), 3089 (C-H benzene), 2967-2853 (C-H aliphatic), 1765 (C=O sydnone), 1729 ( $\mathrm{C}=\mathrm{O}$ ester), 1606-1444 ( $\mathrm{C}=\mathrm{C}$ benzene), 1278, 1107 (C-O ester); ${ }^{1} \mathrm{H}$ NMR $\left(700 \mathrm{MHz}, \mathrm{DMSO}-d_{6}\right) 8.21$ (d, $J=$ $8.52 \mathrm{~Hz}, 2 \mathrm{H}, \mathrm{C} 8-\mathrm{H}, \mathrm{C} 10-\mathrm{H}), 8.08(\mathrm{~d}, J=8.52 \mathrm{~Hz}, 2 \mathrm{H}$, C7-H, C11-H), 7.89 (s, 1 H, C4-H), 3.91 (s, 3 H, C13-H); 
${ }^{13} \mathrm{C}$ NMR (176 MHz, DMSO- $\left.d_{6}\right) 168.88$ (C12), 165.37 (C5), 138.03 (C6), 133.33 (C9), 131.39 (C8, C10), 122.42 (C7, C11), 95.70 (C4), 53.18 (C13). Supplementary data (Spectrum S70-S72).

\section{Synthesis of alcohol sydnone derivatives 47}

3-[4-(Hydroxymethyl)phenyl]sydnone 47: Red needles (95\% ethanol); $52.99 \%$ yield; mp $136-139{ }^{\circ} \mathrm{C}$; IR ( $v_{\max }$, $\left.\mathrm{cm}^{-1}\right) 3391(\mathrm{O}-\mathrm{H}), 3142$ (C-H sydnone), 3094 (C-H benzene), 2935, 2821 ( $\mathrm{C}-\mathrm{H}$ aliphatic), 1711 ( $\mathrm{C}=\mathrm{O}$ sydnone), 1598-1457 ( $\mathrm{C}=\mathrm{C}$ benzene), 1062 ( $\mathrm{C}-\mathrm{O}$ alcohol), 828 (out-of-plane $\mathrm{C}-\mathrm{H}$ bending benzene); ${ }^{1} \mathrm{H}$ NMR (700 MHz, DMSO- $\left.d_{6}\right) 7.89$ (d, $J=8.86 \mathrm{~Hz}, 2 \mathrm{H}, \mathrm{C} 7-\mathrm{H}, \mathrm{C} 11-$ H), $7.76(\mathrm{~s}, 1 \mathrm{H}, \mathrm{C} 4-\mathrm{H}), 7.62(\mathrm{~d}, J=8.52 \mathrm{~Hz}, 2 \mathrm{H}, \mathrm{C} 8-\mathrm{H}$, $\mathrm{C} 10-\mathrm{H}), 5.49(\mathrm{t}, J=5.79 \mathrm{~Hz}, 1 \mathrm{H}, \mathrm{OH}), 4.63(\mathrm{~d}, J=5.79$ $\mathrm{Hz}, 2 \mathrm{H}, \mathrm{C} 12-\mathrm{H}) ;{ }^{13} \mathrm{C}$ NMR (176 MHz, DMSO- $\left.d_{6}\right)$ 168.99 (C5), 147.88 (C6), 133.48 (C9), 128.04 (C7, C11), 121.70 (C8, C10), 95.20 (C4), 62.47 (C12). Supplementary data (Spectrum S73-S75).

\section{Synthesis of aldehyde sydnone derivatives 48}

3-(4-formylphenyl)sydnone 48: Yellow plates (EtOAc); 85.04\% yield; mp $145-149^{\circ} \mathrm{C}$; IR $\left(v_{\max }, \mathrm{cm}^{-1}\right) 3148(\mathrm{C}-\mathrm{H}$ sydnone), 3079 ( $\mathrm{C}-\mathrm{H}$ benzene), 2876 ( $\mathrm{C}-\mathrm{H}$ aldehyde), 1756 ( $\mathrm{C}=\mathrm{O}$ sydnone), 1704 ( $\mathrm{C}=\mathrm{O}$ aldehyde), 1598, 1453 ( $\mathrm{C}=\mathrm{C}$ benzene), 823 (out-of-plane $\mathrm{C}-\mathrm{H}$ benzene bending); ${ }^{1} \mathrm{H}$ NMR (700 MHz, DMSO- $\left.d_{6}\right)$ 10.14 (s, $1 \mathrm{H}, \mathrm{C} 12-$ $\mathrm{H}), 8.22(\mathrm{~d}, J=8.52 \mathrm{~Hz}, 2 \mathrm{H}, \mathrm{C} 8-\mathrm{H}, \mathrm{C} 10-\mathrm{H}), 8.18(\mathrm{~d}, J=$ $8.51 \mathrm{~Hz}, 2 \mathrm{H}, \mathrm{C} 7-\mathrm{H}, \mathrm{C} 11-\mathrm{H}), 7.92(\mathrm{~s}, 1 \mathrm{H}, \mathrm{C} 4-\mathrm{H}) ;{ }^{13} \mathrm{C}$ NMR (176 MHz, DMSO- $d_{6}$ ) 192.70 (C12), 168.89 (C5), 138.95 (C6), 138.58 (C9), 131.53 (C8, C10), 122.91 (C7, C11), 95.86 (C4). Supplementary data (Spectrum S76S78).

\section{Synthesis of 4-acetyl-3-arylsydnones 49-58}

4-Acetyl-3-phenylsydnone 49: Slightly yellow plates (95\% ethanol); $49.04 \%$ yield; $\mathrm{mp} 141-143^{\circ} \mathrm{C}$; IR $\left(v_{\max }, \mathrm{cm}^{-1}\right)$ 3060 (C-H benzene), 1766 (CO sydnone), 1666 (CO acetyl), 1493-1424 ( $\mathrm{C}=\mathrm{C}$ benzene), 1053 (C-CO-C acetyl), 770, 693 (out-of-plane $\mathrm{C}-\mathrm{H}$ bending benzene); ${ }^{1} \mathrm{H}$ NMR $\left(700 \mathrm{MHz}, C D C l_{3}\right) 7.70(\mathrm{t}, J=7.47 \mathrm{~Hz}, 1 \mathrm{H}$, C9-H), 7.61 (t, $J=8.00 \mathrm{~Hz}, 2 \mathrm{H}, \mathrm{C} 7-\mathrm{H}, \mathrm{C} 11-\mathrm{H}), 7.49$ (d, $J=7.49 \mathrm{~Hz}, 2 \mathrm{H}, \mathrm{C} 8-\mathrm{H}, \mathrm{C} 10, \mathrm{H}), 2.54(\mathrm{~s}, 3 \mathrm{H}, \mathrm{C} 13-\mathrm{H})$; ${ }^{13} \mathrm{C}$ NMR (176 MHz, $\mathrm{CDCl}_{3}$ ) 184.23 (C12), 166.27 (C5), 134.94 (C6), 132.37 (C9), 129.44 (C7, C11), 124.87 (C8, C10), 106.06 (C4), 28.12 (C13). Supplementary data (Spectrum S79-S81).

4-Acetyl-3-(4-methylphenyl)sydnone 50: Slightly yellow plates (95\% ethanol); $50.23 \%$ yield; $\mathrm{mp} 118-119{ }^{\circ} \mathrm{C}$; IR $\left(v_{\max }, \mathrm{cm}^{-1}\right) 3060$ (C-H benzene), 2930 (C-H aliphatic), 1783 (CO sydnone), 1678 (CO acetyl), 15091408 ( $\mathrm{C}=\mathrm{C}$ benzene), 1050 (C-CO-C acetyl), 827 (outof-plane $\mathrm{C}-\mathrm{H}$ bending benzene); ${ }^{1} \mathrm{H}$ NMR $(700 \mathrm{MHz}$, $\left.\mathrm{CDCl}_{3}\right) 7.40(\mathrm{~d}, J=8.50 \mathrm{~Hz}, 2 \mathrm{H}, \mathrm{C} 7-\mathrm{H}, \mathrm{C} 11-\mathrm{H}), 7.37(\mathrm{~d}$,
$J=8.52,2 \mathrm{H}, \mathrm{C} 8-\mathrm{H}, \mathrm{C} 10-\mathrm{H}), 2.53(\mathrm{~s}, 3 \mathrm{H}, \mathrm{C} 13-\mathrm{H}), 2.50$ (s, $3 \mathrm{H}, \mathrm{C} 14-\mathrm{H}) ;{ }^{13} \mathrm{C}$ NMR (176 $\left.\mathrm{MHz}, \mathrm{CDCl}_{3}\right) 184.26$ (C12), 166.37 (C5), 143.15 (C9), 132.44 (C6), 129.97 (C7, C11), 124.59 (C8, C10), 105.99 (C4), 28.17 (C13), 21.52 (C14). Supplementary data (Spectrum S82-S84).

4-Acetyl-3-(4-methoxyphenyl)sydnone 51: Slightly yellow plates (95\% ethanol); $64.90 \%$ yield; $\mathrm{mp} 99-101{ }^{\circ} \mathrm{C}$; IR $\left(v_{\max }, \mathrm{cm}^{-1}\right)$ 3080, 3021 (C-H benzene), 2974, 2936 (C-H aliphatic), 1788, 1776 (CO sydnone), 1664 (CO acetyl), 1606, 1590, 1512 ( $\mathrm{C}=\mathrm{C}$ benzene), 1259, 1176 (CO-C alkyl aryl ether), 1051 (C-CO-C acetyl), 833 (outof-plane $\mathrm{C}-\mathrm{H}$ bending benzene); ${ }^{1} \mathrm{H}$ NMR $(700 \mathrm{MHz}$, $\left.\mathrm{CDCl}_{3}\right) 7.43(\mathrm{~d}, J=8.85 \mathrm{~Hz}, 2 \mathrm{H}, \mathrm{C} 7-\mathrm{H}, \mathrm{C} 11-\mathrm{H}), 7.06$ (d, $J=8.86,2 \mathrm{H}, \mathrm{C} 8-\mathrm{H}, \mathrm{C} 10-\mathrm{H}), 3.91(\mathrm{~s}, 3 \mathrm{H}, \mathrm{C} 14-\mathrm{H}), 2.53$ (s, $3 \mathrm{H}, \mathrm{C} 13-\mathrm{H}) ;{ }^{13} \mathrm{C}$ NMR (176 $\left.\mathrm{MHz}, \mathrm{CDCl}_{3}\right) 184.33$ (C12), 166.45 (C5), 162.47 (C9), 127.45 (C6), 126.31 (C7, C11), 114.46 (C8, C10), 105.89 (C4), 55.80 (C14), 28.22 (C13). Supplementary data (Spectrum S85-S87).

4-Acetyl-3-(4-chlorophenyl)sydnone 52: Slightly yellow needles (95\% ethanol); $42.54 \%$ yield; $\mathrm{mp} 133-134{ }^{\circ} \mathrm{C}$; IR $\left(v_{\max }, \mathrm{cm}^{-1}\right)$ 3100, 3062, 3046 (C-H benzene), 1785 (CO sydnone), 1662 (CO acetyl), 1587-1435 ( $\mathrm{C}=\mathrm{C}$ benzene), 1089 (C-Cl), 1049 (C-CO-C acetyl), 837 (out-of-plane C$\mathrm{H}$ bending benzene); ${ }^{1} \mathrm{H}$ NMR $\left(700 \mathrm{MHz}, \mathrm{CDCl}_{3}\right) 7.58$ $(\mathrm{d}, J=8.51 \mathrm{~Hz}, 2 \mathrm{H}, \mathrm{C} 7-\mathrm{H}, \mathrm{C} 11-\mathrm{H}), 7.45$ (d, $J=8.52,2 \mathrm{H}$, $\mathrm{C} 8-\mathrm{H}, \mathrm{C} 10-\mathrm{H}), 2.54$ (s, $3 \mathrm{H}, \mathrm{C} 13-\mathrm{H}) ;{ }^{13} \mathrm{C}$ NMR (176 $\mathrm{MHz}, \mathrm{CDCl}_{3}$ ) 184.32 (C12), 166.03 (C5), 138.86 (C9), 133.20 (C6), 129.76 (C7, C11), 126.27 (C8, C10), 106.01 (C4), 28.12 (C13). Supplementary data (Spectrum S88S90).

4-Acetyl-3-(4-fluorophenyl)sydnone 53: Yellow plates (95\% ethanol); $44.44 \%$ yield; $\mathrm{mp} 91-92{ }^{\circ} \mathrm{C}$; IR ( $v_{\max }$, $\mathrm{cm}^{-1}$ ) 3071, 3020 (C-H benzene), 2970, 2924 (C-H aliphatic), 1826 ( $\mathrm{C}=\mathrm{O}$ sydnone), $1657(\mathrm{C}=\mathrm{O}$ acetyl), 1603, 1513 ( $\mathrm{C}=\mathrm{C}$ benzene), 1053 (C-CO-C acetyl), 1016 (C-F), 843 (out-of-plane $\mathrm{C}-\mathrm{H}$ bending benzene); ${ }^{1} \mathrm{H}$ NMR (700 $\left.\mathrm{MHz}, \mathrm{CDCl}_{3}\right) 7.51$ (dd, $J=8.51,4.59 \mathrm{~Hz}, 2 \mathrm{H}, \mathrm{C} 7-\mathrm{H}$, C11-H), 7.28 (t, $J=8.52,2 \mathrm{H}, \mathrm{C} 8-\mathrm{H}, \mathrm{C} 10-\mathrm{H}), 2.52$ (s, 3 $\mathrm{H}, \mathrm{C} 13-\mathrm{H}) ;{ }^{13} \mathrm{C}$ NMR $\left(176 \mathrm{MHz}, \mathrm{CDCl}_{3}\right) 184.32(\mathrm{C} 12)$, 166.09 (C5) 164.55 (C9), 130.80 (C6), 127.23 (C7, C11), 116.72 (C8, C10), 106.07 (C4), 28.11 (C13). Supplementary data (Spectrum S91-S93).

4-Acetyl-3-(3-nitrophenyl)sydnone 54: Yellow plates (95\% ethanol); $35.17 \%$ yield; $\mathrm{mp} 171-172{ }^{\circ} \mathrm{C}$; IR ( $v_{\max }$, $\mathrm{cm}^{-1}$ ) 3098, 3013 (C-H benzene), 2966-2877 (C-H aliphatic), 1781 ( $\mathrm{C}=\mathrm{O}$ sydnone), $1659(\mathrm{C}=\mathrm{O}$ acetyl), 1538 (asymmetric NO), 1430, $1410 \quad(\mathrm{C}=\mathrm{C}$ benzene), 1343 (symmetric NO), 1052 (C-CO-C acetyl), 810, 743, 680 (out-of-plane $\mathrm{C}-\mathrm{H}$ bending benzene); ${ }^{1} \mathrm{H}$ NMR (700 $\left.\mathrm{MHz}, \mathrm{DMSO}-d_{6}\right) 8.71(\mathrm{t}, J=4.09 \mathrm{~Hz}, 1 \mathrm{H}, \mathrm{C} 7-\mathrm{H}), 8.58$ (dd, $J=8.39,2.15 \mathrm{~Hz}, 1 \mathrm{H}, \mathrm{C} 9-\mathrm{H}), 8.20(\mathrm{~m}, 1 \mathrm{H}, \mathrm{C} 11-\mathrm{H})$, $7.97(\mathrm{t}, J=8.18,1 \mathrm{H}, \mathrm{C} 10-\mathrm{H}), 2.39(\mathrm{~s}, 3 \mathrm{H}, \mathrm{C} 13-\mathrm{H}) ;{ }^{13} \mathrm{C}$ NMR (176 MHz, DMSO- $d_{6}$ ) 184.13 (C12), 166.29 (C5), 148.15 (C8), 136.07 (C6), 132.54 (C11), 131.52 (C10), 
127.36 (C7), 121.80 (C9), 108.09 (C4), 27.89 (C13). Supplementary data (Spectrum S94-S96).

4-Acetyl-3-(4-nitrophenyl)sydnone 55: Yellow plates (95\% ethanol); $35.94 \%$ yield; $\mathrm{mp} 210-212{ }^{\circ} \mathrm{C}$; IR ( $v_{\max }$, $\mathrm{cm}^{-1}$ ) 3097 (C-H benzene), 2990-2866 (C-H aliphatic), $1790(\mathrm{C}=\mathrm{O}$ sydnone), $1670(\mathrm{C}=\mathrm{O}$ acetyl), 1533 (asymmetric $\mathrm{NO}$ ), 1618-1437 ( $\mathrm{C}=\mathrm{C}$ benzene), 1349 (symmetric NO), 1052 (C-CO-C acetyl), 849 (out-of-plane $\mathrm{C}-\mathrm{H}$ bending benzene); ${ }^{1} \mathrm{H}$ NMR $\left(700 \mathrm{MHz}\right.$, DMSO- $\left.d_{6}\right) 8.51$ $(\mathrm{d}, J=8.86 \mathrm{~Hz}, 2 \mathrm{H}, \mathrm{C} 8-\mathrm{H}, \mathrm{C} 10-\mathrm{H}), 8.03(\mathrm{~d}, J=8.86 \mathrm{~Hz}$, $2 \mathrm{H}, \mathrm{C} 7-\mathrm{H}, \mathrm{C} 11-\mathrm{H}), 2.40$ (s, $3 \mathrm{H}, \mathrm{C} 13-\mathrm{H}) ;{ }^{13} \mathrm{C}$ NMR (176 MHz, DMSO- $\left.d_{6}\right) 184.06$ (C12), 166.27 (C5), 149.90 (C9), 139.92 (C6), 127.88 (C7, C11), 125.20 (C8, C10), 108.07 (C4), 27.86 (C13). Supplementary data (Spectrum S97S99).

4-Acetyl-3-(4-methyl-3-nitrophenyl)sydnone 56: Pale yellow plates (95\% ethanol); $23.84 \%$ yield; mp 163-164 ${ }^{\circ} \mathrm{C}$; IR $\left(v_{\max }, \mathrm{cm}^{-1}\right) 3095$ (C-H benzene), 2985-2877 (C$\mathrm{H}$ aliphatic), 1777 ( $\mathrm{C}=\mathrm{O}$ sydnone), 1669 ( $\mathrm{C}=\mathrm{O}$ acetyl), 1532 (asymmetric NO), 1609-1440 ( $\mathrm{C}=\mathrm{C}$ benzene), 1354 (symmetric NO), 1057 (C-CO-C acetyl), 889, 840 (outof-plane $\mathrm{C}-\mathrm{H}$ bending benzene); ${ }^{1} \mathrm{H}$ NMR $(700 \mathrm{MHz}$, DMSO- $\left.d_{6}\right) 8.48(\mathrm{~d}, J=2.15 \mathrm{~Hz}, 1 \mathrm{H}, \mathrm{C} 7-\mathrm{H}), 8.00(\mathrm{dd}, J=$ 8.17, $2.15 \mathrm{~Hz}, 1 \mathrm{H}, \mathrm{C} 11-\mathrm{H}), 7.82(\mathrm{~d}, J=8.39 \mathrm{~Hz}, 1 \mathrm{H}$, C10-H), 2.60 (s, 3 H, C13-H), 2.39 (s, $3 \mathrm{H}, \mathrm{C} 14-\mathrm{H}) ;{ }^{13} \mathrm{C}$ NMR (176 MHz, DMSO-d $\left.d_{6}\right) 184.09$ (C12), 166.31 (C5), 148.83 (C8), 137.87 (C6), 134.32 (C11), 133.76 (C9), 130.54 (C10), 122.57 (C7), 107.87 (C4), 27.95 (C13), 20.14 (C14). Supplementary data (Spectrum S100-S102).

4-Acetyl-3-(4-chloro-3-nitrophenyl)sydnone 57: Yellow plates (95\% ethanol); $19.85 \%$ yield; mp $186{ }^{\circ} \mathrm{C}$; IR ( $v_{\max }$, $\mathrm{cm}^{-1}$ ) 3103, 3084, 3055 (C-H benzene), 2952, 2886 (C-H aliphatic), 1796 ( $\mathrm{C}=\mathrm{O}$ sydnone), 1668 ( $\mathrm{C}=\mathrm{O}$ acetyl), 1542 (asymmetric NO), 1599-1478 ( $\mathrm{C}=\mathrm{C}$ benzene), 1352 (symmetric NO), 1057 (C-CO-C acetyl), 1003 (C-Cl), 893, 838 (out-of-plane $\mathrm{C}-\mathrm{H}$ bending benzene); ${ }^{1} \mathrm{H}$ NMR $\left(700 \mathrm{MHz}, \mathrm{DMSO}-d_{6}\right) 8.64(\mathrm{~d}, J=2.36 \mathrm{~Hz}, 1 \mathrm{H}, \mathrm{C} 7-\mathrm{H})$, $8.14(\mathrm{~d}, J=8.60 \mathrm{~Hz}, 1 \mathrm{H}, \mathrm{C} 10-\mathrm{H}), 8.12(\mathrm{dd}, J=8.60,2.36$ $\mathrm{Hz}, 1 \mathrm{H}, \mathrm{C} 11-\mathrm{H}), 2.39$ (s, $3 \mathrm{H}, \mathrm{C} 13-\mathrm{H}) ;{ }^{13} \mathrm{C}$ NMR (176 $\left.\mathrm{MHz}, \mathrm{DMSO}-d_{6}\right) 184.19$ (C12), 166.13 (C5), 147.53 (C8), 134.43 (C6), 133.48 (C11), 131.55 (C10), 129.74 (C9), 124.07 (C7), 108.02 (C4), 27.87 (C13). Supplementary data (Spectrum S103-S105).

4-Acetyl-3-[4-(methoxycarbonyl)phenyl] sydnone 58: White needles (95\% ethanol); $43.81 \%$ yield; mp 147-149 ${ }^{\circ} \mathrm{C}$; IR $\left(v_{\max }, \mathrm{cm}^{-1}\right) 3107,3009$ (C-H benzene), 29642884 (C-H aliphatic), 1790 ( $\mathrm{C}=\mathrm{O}$ sydnone), $1732(\mathrm{C}=\mathrm{O}$ ester), 1671 ( $\mathrm{C}=\mathrm{O}$ acetyl), 1601-1408 ( $\mathrm{C}=\mathrm{C}$ benzene), 1286, 1109 (C-CO-C ester), 1057 (C-CO-C acetyl), 865 (out-of-plane $\mathrm{C}-\mathrm{H}$ bending benzene); ${ }^{1} \mathrm{H}$ NMR (700 $\left.\mathrm{MHz}, \mathrm{DMSO}-d_{6}\right) 8.29$ (d, $J=8.52 \mathrm{~Hz}, 2 \mathrm{H}, \mathrm{C} 8-\mathrm{H}, \mathrm{C} 10-$ $\mathrm{H}), 7.59$ (d, $J=8.52 \mathrm{~Hz}, 2 \mathrm{H}, \mathrm{C} 7-\mathrm{H}, \mathrm{C} 11-\mathrm{H}), 4.01(\mathrm{~s}, 3$ $\mathrm{H}, \mathrm{C} 15-\mathrm{H}), 2.56$ (s, $3 \mathrm{H}, \mathrm{C} 13-\mathrm{H}) ;{ }^{13} \mathrm{C}$ NMR $(176 \mathrm{MHz}$, DMSO- $\left.d_{6}\right) 184.27$ (C12), 165.94 (C14), 165.21 (C5),
138.03 (C6), 133.90 (C9), 130.75 (C8, C10), 125.13 (C7, C11), 106.11 (C4), 52.82 (C15), 28.07 (C13). Supplementary data (Spectrum S106-S108).

\section{Synthesis of bis-sydnone styryl ketones 61-63}

3-Phenyl-4-\{1-oxo-3-[4-(sydnon-3-yl]-2-propenyl\} sydnone 61: Yellow plates; $25.32 \%$ yield; $\mathrm{mp} 244{ }^{\circ} \mathrm{C}$; IR $\left(v_{\max }, \mathrm{cm}^{-1}\right) 3133$ (C-H sydnone), 3105 (C-H styryl), 3070 ( $\mathrm{C}-\mathrm{H}$ benzene), $1778(\mathrm{C}=\mathrm{O}$ sydnone), $1754(\mathrm{C}=\mathrm{O}$ sydnone), 1655 ( $\mathrm{C}=\mathrm{O}$ styrylketone), 1599 ( $\mathrm{C}=\mathrm{C}$ styryl), 1588-1422 ( $\mathrm{C}=\mathrm{C}$ benzene), 1030 (out-of-plane $\mathrm{C}-\mathrm{H}$ bending styryl), 835, 765, 695 (out-of-plane $\mathrm{C}-\mathrm{H}$ bending benzene); ${ }^{1} \mathrm{H}$ NMR $\left(700 \mathrm{MHz}, \mathrm{DMSO}-d_{6}\right) 8.04(\mathrm{~d}, J=$ $8.51 \mathrm{~Hz}, 2 \mathrm{H}, \mathrm{C} 7-\mathrm{H}, \mathrm{C} 11-\mathrm{H}), 7.99(\mathrm{~d}, J=8.51 \mathrm{~Hz}, 2 \mathrm{H}$, C10-H, C8-H), 7.85 (s, 1H, C4-H), 7.81-7.74 (m, 4H, C12-H, C13-H, C7' -H, C11' -H), 7.68 (t, $J=7.67 \mathrm{~Hz}, 2 \mathrm{H}$, $\left.\mathrm{C} 10^{\prime}-\mathrm{H}, \mathrm{C} 8^{\prime}-\mathrm{H}\right), 7.81-7.74(\mathrm{~m}, 1 \mathrm{H}, \mathrm{C} 9-\mathrm{H}) ;{ }^{13} \mathrm{C}$ NMR (176 MHz, DMSO- $d_{6}$ ) 174.73 (C14), 168.92 (C5), 166.29 (C5'), 141.49 (C12), 138.39 (C9), 135.95 (C6/C6'), 135.70 (C6/C6'), 132.57 (C9'), 130.42 (C8', C10'), 129.77 (C8, C10), 125.93 (C7', C11'), 124.85 (C13), 122.72 (C7, C11), 108.35 (C4'), 95.39 (C4); HRMS m/z $[\mathrm{M}+\mathrm{Na}]^{+} 399.0700 \quad \mathrm{C}_{19} \mathrm{H}_{12} \mathrm{~N}_{4} \mathrm{O}_{5} \mathrm{Na} \quad$ (calcd. 399.0696). Supplementary data (Spectrum S109-S112).

3-(4-Methylphenyl)-4-\{1-oxo-3-[4-(sydnon-3-yl)

phenyl]-2-propenyl\} sydnone 62: Yellow plates; $18.4 \%$ yield; mp $210-212{ }^{\circ} \mathrm{C}$; IR $\left(v_{\max }, \mathrm{cm}^{-1}\right) 3171(\mathrm{C}-\mathrm{H}$ sydnone), 3144 (C-H styryl), 3091-3065 (C-H benzene), $1787(\mathrm{C}=\mathrm{O}$ sydnone), 1765 ( $\mathrm{C}=\mathrm{O}$ sydnone), $1658(\mathrm{C}=\mathrm{O}$ styrylketone), 1605 ( $\mathrm{C}=\mathrm{C}$ styryl), $1520-1425(\mathrm{C}=\mathrm{C}$ benzene), 1040 (out-of-plane $\mathrm{C}-\mathrm{H}$ bending styryl), 825 (outof-plane $\mathrm{C}-\mathrm{H}$ bending benzene); ${ }^{1} \mathrm{H}$ NMR $(700 \mathrm{MHz}$, DMSO- $\left.d_{6}\right) 8.19(\mathrm{~d}, J=8.51 \mathrm{~Hz}, 2 \mathrm{H}, \mathrm{C} 7-\mathrm{H}, \mathrm{C} 11-\mathrm{H}), 7.99$ (d, $J=8.86 \mathrm{~Hz}, 2 \mathrm{H}, \mathrm{C} 10-\mathrm{H}, \mathrm{C} 8-\mathrm{H}), 7.85(\mathrm{~s}, 1 \mathrm{H}, \mathrm{C} 4-\mathrm{H})$, $7.83(\mathrm{~d}, J=16.01 \mathrm{~Hz}, 1 \mathrm{H}, \mathrm{C} 12-\mathrm{H}), 7.74(\mathrm{~d}, J=16.00 \mathrm{~Hz}$, $1 \mathrm{H}, \mathrm{C} 13-\mathrm{H}), 7.64\left(\mathrm{~d}, J=8.17 \mathrm{~Hz}, 2 \mathrm{H}, \mathrm{C} 7{ }^{\prime}-\mathrm{H}, \mathrm{C} 11^{\prime}-\mathrm{H}\right)$, $7.48\left(\mathrm{~d}, J=8.18 \mathrm{~Hz}, 2 \mathrm{H}, \mathrm{C} 10^{\prime}-\mathrm{H}, \mathrm{C} 8^{\prime}-\mathrm{H}\right), 2.46(\mathrm{~s}, 3 \mathrm{H}$, $\left.\mathrm{CH}_{3}\right) ;{ }^{13} \mathrm{C}$ NMR $\left(176 \mathrm{MHz}, \mathrm{DMSO}-d_{6}\right) 174.74(\mathrm{C} 14)$, 168.92 (C5), 166.34 (C5'), 142.76 (C12), 141.38 (C9'), 138.40 (C9), 135.93 (C6), 133.23 (C6'), 130.40 (C8', C10), 130.11 (C8, C10), 125.67 (C7', C11'), 124.97 (C13), 122.71 (C7, C11), 108.23 (C4'), 95.39 (C4), 21.42 $\left(\mathrm{CH}_{3}\right) ;$ HRMS $m / z[\mathrm{M}+\mathrm{Na}]^{+} 413.0856 \mathrm{C}_{20} \mathrm{H}_{14} \mathrm{~N}_{4} \mathrm{O}_{5} \mathrm{Na}$ (calcd. 413.0857). Supplementary data (Spectrum S113S116).

3-(4-Chlorophenyl)-4-\{1-oxo-3-[4-(sydnon-3-yl)

phenyl]-2-propenyl\} sydnone 63: Yellow plates; $24.95 \%$ yield; mp $198-199{ }^{\circ} \mathrm{C}$; IR $\left(v_{\max }, \mathrm{cm}^{-1}\right) 3146(\mathrm{C}-\mathrm{H}$ sydnone), 3121 (C-H styryl), 3075-3061 (C-H benzene), 1777 ( $\mathrm{C}=\mathrm{O}$ sydnone), 1739 ( $\mathrm{C}=\mathrm{O}$ sydnone), $1659(\mathrm{C}=\mathrm{O}$ styrylketone), 1598 ( $\mathrm{C}=\mathrm{C}$ styryl), 1520-1431 ( $\mathrm{C}=\mathrm{C}$ benzene), 1033 (out-of-plane $\mathrm{C}-\mathrm{H}$ bending styryl), 839 (outof-plane $\mathrm{C}-\mathrm{H}$ bending benzene); ${ }^{1} \mathrm{H}$ NMR $(700 \mathrm{MHz}$, DMSO- $\left.d_{6}\right) 8.05$ (d, $\left.J=8.86 \mathrm{~Hz}, 2 \mathrm{H}, \mathrm{C} 7-\mathrm{H}, \mathrm{C} 11-\mathrm{H}\right), 8.01$ 
(d, $J=8.52 \mathrm{~Hz}, 2 \mathrm{H}, \mathrm{C} 10-\mathrm{H}, \mathrm{C} 8-\mathrm{H}), 7.85(\mathrm{~s}, 1 \mathrm{H}, \mathrm{C} 4-\mathrm{H})$, $7.84(\mathrm{~d}, J=16.01 \mathrm{~Hz}, 1 \mathrm{H}, \mathrm{C} 12-\mathrm{H}), 7.77(\mathrm{~d}, J=15.67 \mathrm{~Hz}$, 1H, C13-H), 7.82 (d, $\left.J=8.86 \mathrm{~Hz}, 2 \mathrm{H}, \mathrm{C} 7^{\prime}-\mathrm{H}, \mathrm{C} 11^{\prime}-\mathrm{H}\right)$, 7.79 (d, $\left.J=8.85 \mathrm{~Hz}, 2 \mathrm{H}, \mathrm{C} 10^{\prime}-\mathrm{H}, \mathrm{C} 8^{\prime}-\mathrm{H}\right) ;{ }^{13} \mathrm{C}$ NMR $(176$ $\left.\mathrm{MHz}, \mathrm{DMSO}-d_{6}\right) 174.76$ (C14), 168.92 (C5), 166.18 (C5'), 141.70 (C12), 138.33 (C9), 137.35 (C9'), 136.00 (C6), 134.43 (C6'), 130.47 (C8, C10), 129.92 (C7', C11'), 127.96 (C8', C10'), 124.68 (C13), 122.74 (C7, C11), 108.56 (C4'), 95.41 (C4); HRMS $m / z[\mathrm{M}+\mathrm{Na}]^{+} 433.0310$ $\mathrm{C}_{19} \mathrm{H}_{11} \mathrm{ClN}_{4} \mathrm{O}_{5} \mathrm{Na}$ (calcd. 433.0313). Supplementary data (Spectrum S117-S120).

\section{Biological evaluation (in vitro cyclooxygenase inhibitory assay)}

The in vitro cyclooxygenase (COX-1/COX-2) inhibitory assay was performed for all sydnone (37-58) and bissydnone styryl ketone derivatives (61-63) and presented in Table 1. The two reference drugs were SC-560 as a selective COX-1 inhibitor [19] and DuP-697 as a selective COX-2 inhibitor [20]. Both 3-arylsydnone and 4acetyl-3-arylsydone showed moderate to low inhibitory activity against both COX isozymes. The calculated selectivity indices ranged from 0.29 to 1.27 . Consequently, none of the compounds $37-56$ can be considered a promising selective or non-selective COX inhibitor. Nevertheless, the findings of the study disclosed that acetylation of the sydnone ring at C-4 had increased the inhibitory activity of most of the tested compounds against COX enzymes. For example, 51, 54, 55, 57 and 58 inhibited more than $50 \%$ of at least one COX isoform while their non-acetylated analogues exhibited inhibitory activities in the range of $20-40 \%$. Therefore, it can be speculated that the presence of an acetyl group at C-4 contributes in the interaction of the sydnone ring with COX active site. Noticeably, compound 63 at a concentration of $200 \mu \mathrm{M}$ was the most potent COX-2 inhibitor suppressing $89.45 \%$ of the enzyme activity. On the other hand, it inhibited only $33 \%$ of COX-1 activity. Consequently, the calculated selectivity index of $\mathbf{6 3}$ was 2.70 . On the contrary, $\mathbf{6 1}$ and $\mathbf{6 2}$ were almost inactive against COX- 2 while they inhibited $44 \%$ and $73 \%$ of COX-1, respectively. The results thus revealed the importance of combining two sydnone molecules via a styryl ketone linker as a new selective COX-2 inhibitor, provided that the terminal phenyl ring bears an electron-withdrawing group like a chlorine atom.

\section{Molecular docking analysis}

Findings of the current study showed that the extra precision docking scores of 37-58 and 61-63 with COX-2 ranged from -4.134 to -1.384 , and the binding energy MM-GBSA ranged from -43.827 to $-5.336 \mathrm{kcal} / \mathrm{mol}$ (Table 2). Apparently, the acetylated sydnones 49-58 exhibited a lower binding energy than their non- acetylated counterparts revealing a more stable binding of 4-acetyl-3-arylsydnone with COX-2. Consequently, the existence of the acetyl group at the carbon C-4 of the sydnone ring is important for COX-2 inhibitory activity. Compound 63 had the lowest MM-GBSA value which came in accordance with its potent in vitro COX2 inhibitory activity. In contrast, the inactive sydnone analogues against COX-2 like 37, 41 and 62 ranked high in terms of the binding energy with COX-2.

On the other hand, the docking scores of the studied compounds with the active site of COX-1 were -5.524 to -1.694 and the calculated MM-GBSA ranged from 30.664 to $-1.831 \mathrm{kcal} / \mathrm{mol}$ as shown in Table 2 . Except for compounds 49, the acetylated sydnones had a lower binding energy than that of the non-acetylated ones suggesting a high affinity of 4-acetyl-3-arylsydnone to COX1. Our finding displayed a strong correlation $\left(r^{2}=0.83\right)$ between the calculated MM-GBSA and the in vitro COX-2 inhibition by AChE ELISA, while docking scores were not well correlated to the inhibitory activity (Fig. 5a). Likewise, a moderate linear correlation $\left(r^{2}=0.63\right)$ was observed between MM-GBSA and the in vitro activity against COX-1 (Fig. 5b).

Analysis of the binding poses disclosed that the sydnones of the present study oriented similarly in the COX-2 binding cavity; i.e. binding with residues from the MBD and the CD (Tables 3 and 4). Moreover, the binding modes were close to that of celecoxib, especially the interaction with Arg513 which is pivotal for selective COX-2 inhibition. However, celecoxib binds noncovalently with Arg513 through positive charge interaction between the sulphonamide and guanidinium. Sydnones interact with Arg513 via different types of noncovalent interactions like positive charged, $\pi-\pi, \pi-$ cation and salt-bridging. Access of celecoxib and sydnones to the adjunct pocket were through hydrophobic interaction with Tyr91. Other types of interactions like polar, hydrogen bonding and negative/positive charges with other residues in the binding site were also involved. Interestingly, the acetyl function at $\mathrm{C}-4$ of the sydnone ring contributed as a hydrogen bond acceptor through its carbonyl oxygen atom with residues at the top of the COX-2 binding site.

Compound 63 (bis-sydnone styryl ketone) had the lowest MM-GBSA value $(-34.763 \mathrm{kcal} / \mathrm{mol})$ with a considerable low docking score with COX-2 suggesting a good binding pose and a stable ligand-enzyme complex as displayed in Fig. 6a and supplementary data S121, Tables 3 and 4 . It was found that one sydnone ring and the styryl ketone moiety bound at the MBD (residues 75115) and the mouth of the catalytic region, while the other sydnone and the terminal benzene fit inside the catalytic domain. At the MBD and the mouth of the catalytic domain, the following interactions were also 


\begin{tabular}{|c|c|c|c|c|c|c|}
\hline Compound & R1 & $\mathbf{R 2}$ & $\mathbf{R 3}$ & $\begin{array}{l}\text { COX-1 } \\
\text { inhibition (\%) }\end{array}$ & $\begin{array}{l}\text { COX-2 } \\
\text { inhibition (\%) }\end{array}$ & SI-2 \\
\hline 37 & $\mathrm{H}$ & $\mathrm{H}$ & $\mathrm{H}$ & 47.17 & 14.76 & 0.31 \\
\hline 38 & $\mathrm{CH}_{3}$ & $\mathrm{H}$ & $\mathrm{H}$ & 34.4 & 26.09 & 0.76 \\
\hline 39 & $\mathrm{OCH}_{3}$ & $\mathrm{H}$ & $\mathrm{H}$ & 36.07 & 39.68 & 1.10 \\
\hline 40 & $\mathrm{Cl}$ & $\mathrm{H}$ & $\mathrm{H}$ & 22.01 & 16.2 & 0.74 \\
\hline 41 & $\mathrm{~F}$ & $\mathrm{H}$ & $\mathrm{H}$ & 39.88 & 15.99 & 0.40 \\
\hline 42 & $\mathrm{H}$ & $\mathrm{NO}_{2}$ & $\mathrm{H}$ & 37.32 & 39.21 & 1.05 \\
\hline 43 & $\mathrm{NO}_{2}$ & $\mathrm{H}$ & $\mathrm{H}$ & 33.1 & 20.11 & 0.61 \\
\hline 44 & $\mathrm{CH}_{3}$ & $\mathrm{NO}_{2}$ & $\mathrm{H}$ & 30.87 & 23.97 & 0.78 \\
\hline 45 & $\mathrm{Cl}$ & $\mathrm{NO}_{2}$ & $\mathrm{H}$ & 37.98 & 23.06 & 0.61 \\
\hline 46 & $\mathrm{CH}_{3} \mathrm{COO}$ & $\mathrm{H}$ & $\mathrm{H}$ & 31.53 & 29.17 & 0.93 \\
\hline 47 & $\mathrm{CH}_{2} \mathrm{OH}$ & $\mathrm{H}$ & $\mathrm{H}$ & 90 & 61.79 & 0.69 \\
\hline 48 & $\mathrm{CHO}$ & $\mathrm{H}$ & $\mathrm{H}$ & 49.94 & 43.89 & 0.88 \\
\hline 49 & $\mathrm{H}$ & $\mathrm{H}$ & $\mathrm{COCH}_{3}$ & 44.12 & 29.65 & 0.67 \\
\hline 50 & $\mathrm{CH}_{3}$ & $\mathrm{H}$ & $\mathrm{COCH}_{3}$ & 61.71 & 18.9 & 0.31 \\
\hline 51 & $\mathrm{OCH}_{3}$ & $\mathrm{H}$ & $\mathrm{COCH}_{3}$ & 44.55 & 56.67 & 1.27 \\
\hline 52 & $\mathrm{Cl}$ & $\mathrm{H}$ & $\mathrm{COCH}_{3}$ & 36.88 & 17.88 & 0.48 \\
\hline 53 & $\mathrm{~F}$ & $\mathrm{H}$ & $\mathrm{COCH}_{3}$ & 46.67 & 23.8 & 0.51 \\
\hline 54 & $\mathrm{H}$ & $\mathrm{NO}_{2}$ & $\mathrm{COCH}_{3}$ & 57.76 & 36.39 & 0.63 \\
\hline 55 & $\mathrm{NO}_{2}$ & $\mathrm{H}$ & $\mathrm{COCH}_{3}$ & 77.65 & 22.71 & 0.29 \\
\hline 56 & $\mathrm{CH}_{3}$ & $\mathrm{NO}_{2}$ & $\mathrm{COCH}_{3}$ & 37.19 & 45.17 & 1.21 \\
\hline 57 & $\mathrm{Cl}$ & $\mathrm{NO}_{2}$ & $\mathrm{COCH}_{3}$ & 42.1 & 51.99 & 1.23 \\
\hline 58 & $\mathrm{CH}_{3} \mathrm{COO}$ & $\mathrm{H}$ & $\mathrm{COCH}_{3}$ & 47.44 & 57.12 & 1.20 \\
\hline 61 & $\mathrm{H}$ & $\mathrm{H}$ & & 44.05 & 25.04 & 0.57 \\
\hline 62 & $\mathrm{CH}_{3}$ & $\mathrm{H}$ & & 73.1 & 16.5 & 0.23 \\
\hline 63 & $\mathrm{Cl}$ & $\mathrm{H}$ & & 33.17 & 89.45 & 2.70 \\
\hline $\mathrm{SC}-560(20 \mathrm{nM})$ & - & - & & 53.12 & 18.12 & 0.34 \\
\hline DuP-697 (5 nM) & - & - & & 17.2 & 56.67 & 3.29 \\
\hline
\end{tabular}

SI-2: COX-2 selectivity index. The final concentration of the study compounds in the COX reaction tubes was $200 \mu \mathrm{M}$. Standard drugs (SC-560 and DuP-697) were used at their recommended $\mathrm{IC}_{50}$ values

observed: Tyr91 had a hydrophobic interaction and faceto-face $\pi-\pi$ interaction with the benzene ring, edge-toface $\pi-\pi$ interaction with the sydnone ring, and $\pi$-cation interaction with sydnone N-3 atom. The carbonyl of the styryl ketone had polar interactions with both Asn87 and Thr88. In the catalytic domain, $\mathbf{6 3}$ had the following interactions: The benzene had $\pi$-cation interaction with Lys511 and hydrophobic interactions with Pro512 and Pro514, and the sydnone ring had a positively charged interaction with Arg513. The chlorine atom had a negatively charged interaction with Glu510 and Glu520 at the core of the catalytic site and a hydrophobic 
Table 2 Extra precision (XP) binding scores and molecular mechanics based on generalised born/surface area (MM-GBSA) for docking poses of $\mathbf{3 7 - 5 8}$ and $\mathbf{6 1 - 6 3}$ into cyclooxygenase enzymes

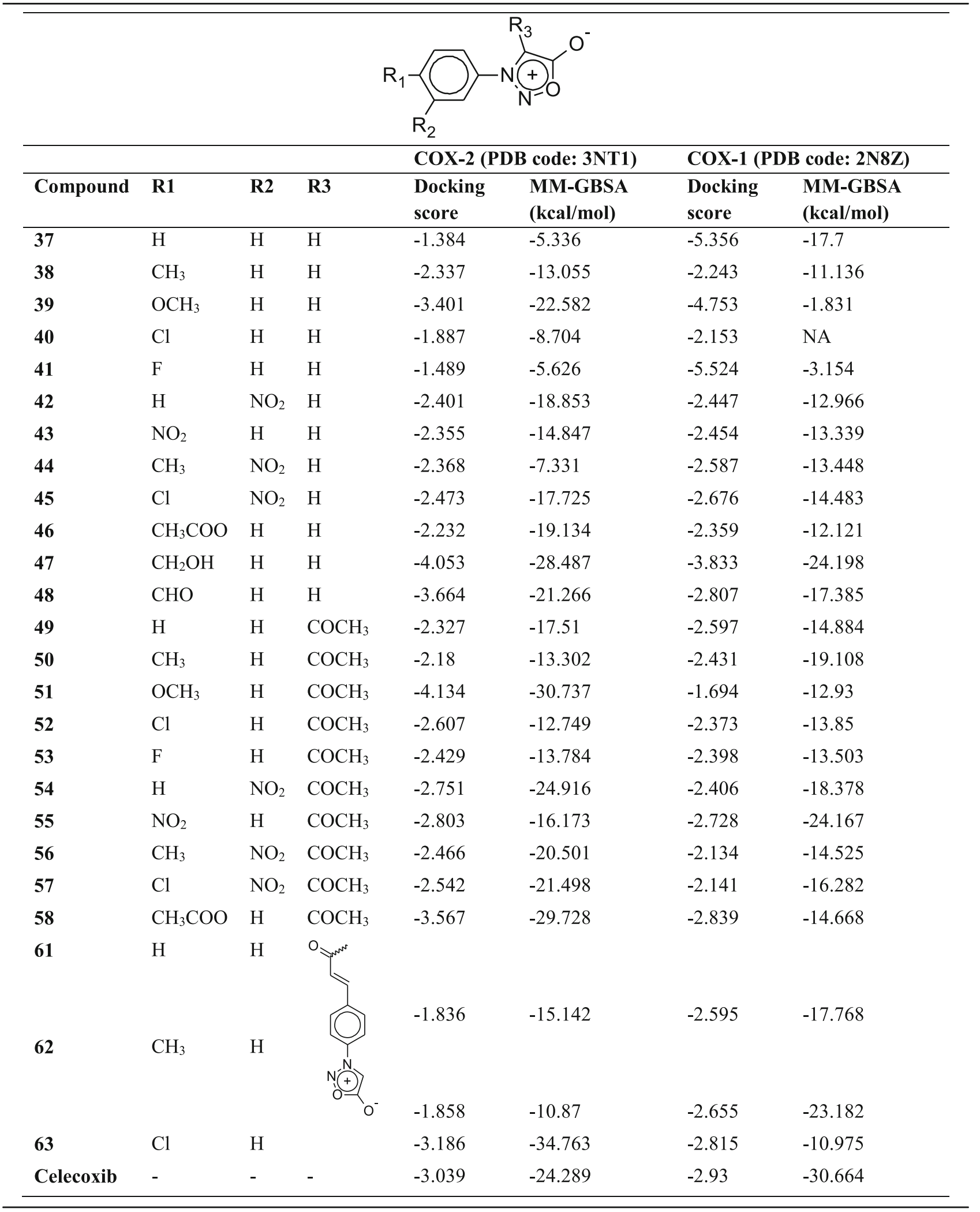




\section{cox-2 inhibition (\%)}
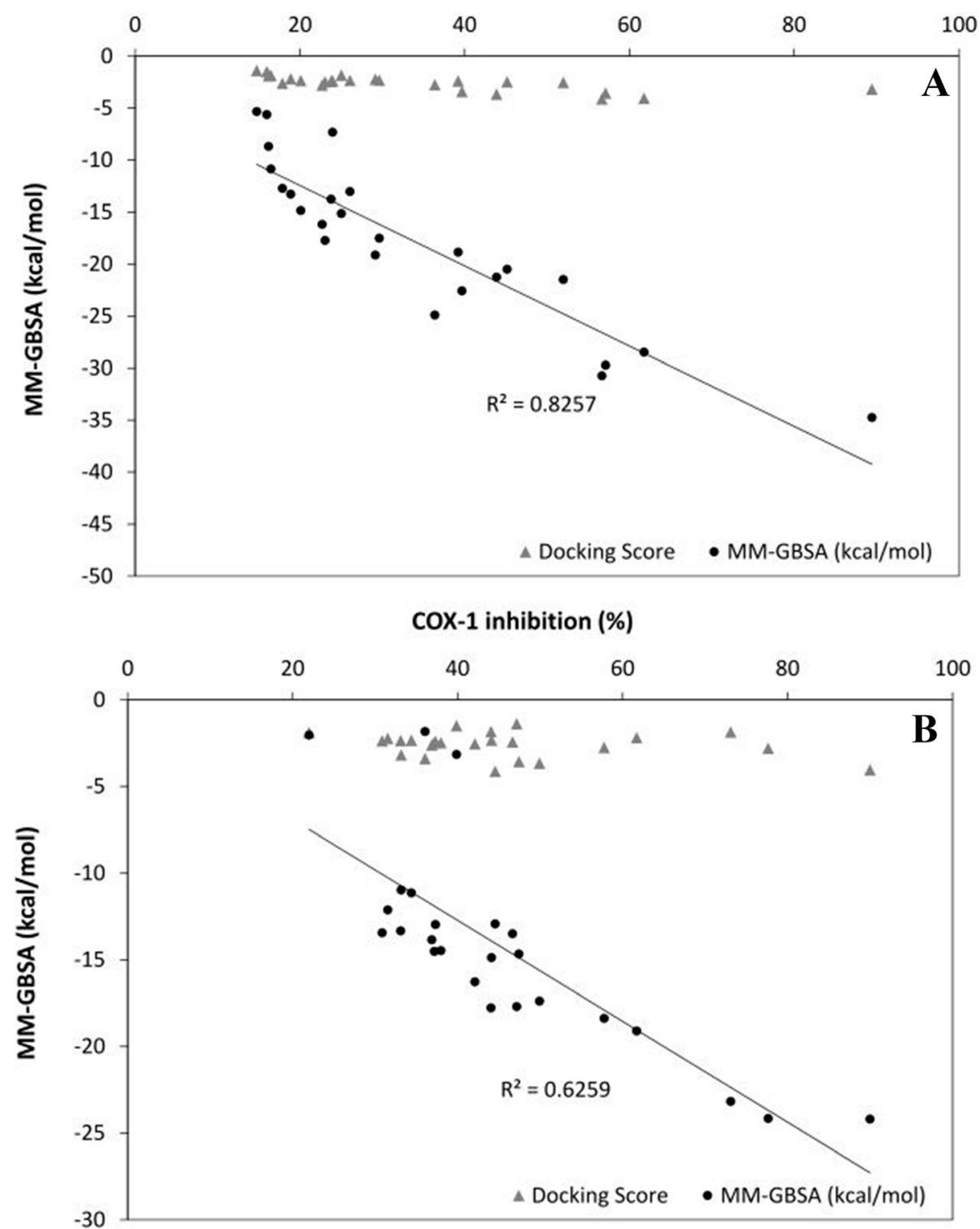

Fig. 5 The calculated binding free energy (MM-GBSA)/Glide docking scores vs. the in vitro inhibitory activity (\%) of compounds 37-63 against $\operatorname{COX}-2(\mathbf{a})$ and $\operatorname{COX}-1(\mathbf{b})$

interaction with the side pocket residue Tyr475 (Fig. 6a and supplementary data S121). Likewise, 4-acetyl-3-(4methoxyphenyl)sydnone 51 and 4-acetyl-3-[4-(methoxycarbonyl) phenyl]sydnone $\mathbf{5 8}$ had remarkably low MMGBSA values of -30.737 and $-29.728 \mathrm{kcal} / \mathrm{mol}$ (Table 2) with binding poses like that of compound 63 . However, compound $\mathbf{5 1}$ binds essentially at the base of the catalytic domain with fewer interactions inside the active site (Fig. 6b and supplementary data S121).

The binding pose of celecoxib as a selective COX-2 inhibitor was also investigated (Fig. 6c, Tables 3 and 4). The sulphonamide group of celecoxib had hydrogen bonds with the side chain of Asn87 and the carbonyl function of Lys511. The methyl group and benzene rings had hydrophobic interactions with Tyr91 of the MBD and Tyr475, Pro512 and Pro514 of the catalytic domain. Moreover, positively charged interactions with Lys511 and Arg513 were also observed. The residues Glu480, Glu510 and Glu520 had negatively charged interactions. Many residues at the MBD such as Asn87, His90, Thr94 and His95 were also involved in polar interactions with celecoxib atoms.

Analysis of the binding poses of sydnones with COX-1 revealed varied binding hits. Compounds 37-41 had a 
Table 3 The interactions of sydnones $\mathbf{3 7 - 5 8}$ and $\mathbf{6 1 - 6 3}$ and celecoxib with Arg513 of COX-2 binding site

\begin{tabular}{|c|c|c|}
\hline Compound & Interaction type & Distance $(\AA ̊)$ \\
\hline 37 & $\pi$-cation & 5.91 \\
\hline 38 & Positive charge & NA \\
\hline 39 & Positive charge & NA \\
\hline 40 & Positive charge & NA \\
\hline 41 & $\pi$-cation & 6.02 \\
\hline 42 & $\pi$-cation, $\pi-\pi$ stacking & $5.76,5.38$ \\
\hline 43 & $\pi$-cation & 6.39 \\
\hline 44 & $\pi$-cation, $\pi-\pi$ stacking & $5.75,5.36$ \\
\hline 45 & $\pi$-cation & 6.01 \\
\hline 46 & Positive charge & NA \\
\hline 47 & $\pi$-cation & 6.48 \\
\hline 48 & $\pi$-cation & 6.21 \\
\hline 49 & Positive charge & NA \\
\hline 50 & Positive charge, hydrogen bond & NA, 3.09 \\
\hline 51 & Positive charge, hydrogen bond & NA, 2.68 \\
\hline 52 & Positive charge, hydrogen bond & NA, 3.07 \\
\hline 53 & Positive charge, hydrogen bond & NA, 3.08 \\
\hline 54 & Positive charge, hydrogen bond & NA, 2.62 \\
\hline 55 & $\pi$-cation & 6.35 \\
\hline 56 & salt bridge & 4.75 \\
\hline 57 & salt bridge & 4.50 \\
\hline 58 & $\pi$-cation & 6.52 \\
\hline 61 & $\pi$-cation & 6.01 \\
\hline 62 & $\pi$-cation & 6.04 \\
\hline 63 & Positive charge & NA \\
\hline Celecoxib & Positive charge & NA \\
\hline
\end{tabular}

NA not available

high number of hydrophobic interactions particularly in the apex of the catalytic loop (Trp387, Tyr385). The existence of a nitro group at the para position of the phenyl ring as in compound 43 resulted in an extra hydrogen bond. In contrast to COX-2, the free energy calculation showed that the complexes of compounds 63 and 51 with COX-1 had high MM-GBSA values of 10.975 and $-12.930 \mathrm{kcal} / \mathrm{mol}$, respectively. This indicates an unstable complex with the enzyme. Taking a closer look into the binding pose of $\mathbf{6 3}$ with COX-1 revealed that its orientation inside the binding site of COX-1 is an antipode to its binding with COX-2 (Fig. 7a). The chlorine atom oriented towards the MBD, whereas the styryl ketone moiety was located inside the catalytic domain. Of interest, 51 had a binding free energy value of $-12.93 \mathrm{kcal} / \mathrm{mol}$ with a relatively high docking score of -1.694 which means it did not fit well inside the binding pocket of COX1. As shown in Fig. 7b, the carbonyl of the acetyl group had a hydrogen bonding with Ser87. The residue Lys511 of the catalytic region had a $\pi$-cation interaction with the phenyl ring and a positively charged interaction with $\mathrm{N}-3$ of the sydnone ring. Hydrophobic interactions (Cys512 and Pro514), polar interactions (Ser87 and His513) and positively charged interactions (Glu520) were also observed. The low docking scores revealed that these interactions are disfavoured.

\section{Discussion}

Structure elucidation of compounds 61-63

Claisen-Schmidt's condensation is an aldol condensation where an aldehyde/ketone possessing an $\alpha$ hydrogen reacts with another carbonyl compound lacking the $\alpha$ hydrogen to produce an $\alpha, \beta$-unsaturated product. The condensation usually requires the presence of a strong base as a catalyst, even though some reported a successful acid-catalysed condensation [21]. In the current research, we applied the base-catalysed aldol condensation approaches to join the 4-acetylated sydnones (49, 50 and 52) with the aldehyde derivative $\mathbf{4 8}$ in order to produce bis-sydnone styryl ketone molecules. Consequently, three compounds 61-63 were successfully synthesized, purified and characterized. Because 61-63 were considered to be novel molecules, a comprehensive structural elucidation was performed using FTIR, ${ }^{1} \mathrm{H}$ NMR, ${ }^{13} \mathrm{C}$ NMR and MS spectra. To make the peak-atom assignments, the obtained spectral data was compared to the most similar analogues found in the literature like phenyl styryl ketones [22, 23] and mono-sydnone styryl ketones [13, 24].

The FTIR data was summarized in Table 5 and supplementary data (S109, S113 and S117). Interestingly, the appearance of three strong bands in the carbonyl region delineated the existence of three carbonyl groups owing to the two sydnones and the styryl ketone. The first two bands $\left(\approx 1780\right.$ and $\left.1760-1740 \mathrm{~cm}^{-1}\right)$ distinctive of the sydnone carbonyl were at high wavenumbers due to the sydnone ring's strain [1]. The styryl carbonyl was shifted to a lower frequency due to the conjugation with the $\pi$ electron systems of the styryl and the sydnone [22, 23]. Further structural confirmation was made on the basis of $s p^{2} \mathrm{C}-\mathrm{H}$ stretches of the sydnone and styryl moieties which occurred within their expected ranges. The sydnone $\mathrm{C} 4-\mathrm{H}$ appeared at high wavenumbers (3170$3100 \mathrm{~cm}^{-1}$ ) while the styryl $\mathrm{C}-\mathrm{H}$ appeared about 3100 $\mathrm{cm}^{-1}$. Because the out-of-plane bending of the $s p^{2} \mathrm{C}-\mathrm{H}$ is of great importance for determining the substitution pattern of the benzene ring and the styryl compounds, they were well examined. First, the strong band about $1030 \mathrm{~cm}^{-1}$ indicated the formation of trans substituted styryl double bonds [23]. Second, the appearance of one band in the range of $850-800 \mathrm{~cm}^{-1}$ revealed that all 
Table 4 Hydrogen bonding and hydrophobic interactions of sydnones $\mathbf{3 7 - 5 8}$ and $\mathbf{6 1 - 6 3}$ and celecoxib with COX-2 binding site

\begin{tabular}{|c|c|c|c|}
\hline \multirow[t]{2}{*}{ Compound } & \multicolumn{2}{|c|}{ Hydrogen bonding } & \multirow[t]{2}{*}{ Hydrophobic interactions } \\
\hline & Residue & Distance $(\AA ̊)$ & \\
\hline 37 & Glu520 & 1.91 & Tyr475, Pro512 \\
\hline 38 & Glu520 & 3.11 & Tyr91, Tyr475, Pro512, Pro514 \\
\hline 39 & His90, Lys511 & $1.95,2,14$ & Tyr91, Pro514 \\
\hline 40 & Glu520 & 2.69 & Tyr91, Tyr475, Pro512, Pro514 \\
\hline 41 & Glu529 & 2.23 & Tyr475, Pro512, Pro514 \\
\hline 42 & His90, Glu520 & $3.12,2.23$ & Tyr91, PTyr475, Pro512, Pr0514 \\
\hline 43 & non & non & Tyr91, Tyr475, Pro512, Pro514 \\
\hline 44 & His90, Glu520 & $2.95,2.21$ & Tyr91, Tyr475, Pro512, Pro514 \\
\hline 45 & His90, Glu521 & $3,32,2.23$ & Tyr91, Tyr475, Pro512, Pro514 \\
\hline 46 & His90, Lys511 & $2.39,2.03$ & Tyr91, Tyy475, Pro514 \\
\hline 47 & Lys511, Glu520 & $1.73,1.98$ & Tyr475, Pro512, Pro514 \\
\hline 48 & Glu520 & 1.93 & Tyr91, Tyr475, Pro512, Pro514 \\
\hline 49 & Lys511, Glu520 & $2.03,3.42$ & Tyr91, Tyr475, Pro514 \\
\hline 50 & Lys511, Arg513 & $1.89,3.09$ & Tyr91, Tyr475, Pro514 \\
\hline 51 & Lys511, Arg513 & $1.89,2.68$ & Tyr91, Pro512, Pro514 \\
\hline 52 & Lys511, Arg513 & $1.95,3.07$ & Tyr91, Tyr475, Pro514 \\
\hline 53 & Lys511, Arg513 & $1.90,3.08$ & Tyr91, Tyr475, Pro515 \\
\hline 54 & Arg513, Glu520 & $2.62,3.33$ & Tyr91, Pro512, Pro514 \\
\hline 55 & Lys511, Glu520 & $2.13,2.20$ & Tyr475, Pro512, Pro514 \\
\hline 56 & His90, Lys511 & $2.58,2.06$ & Tyr91, Tyr475, Pro514 \\
\hline 57 & His90, Lys511 & $2.47,2.12$ & Tyr91, tyr475, Pro514 \\
\hline 58 & Glu520 & 2.23 & Tyr91, Tyr475, Leu481, Pro512, Pro514 \\
\hline 61 & Glu520 & 2.19 & Tyr91, Tyr475, Pro512, Pro514 \\
\hline 62 & Glu520 & 2.23 & Tyr91, Tyr475, Pro512, Pro514 \\
\hline 63 & non & non & Tyr91, Tyr475, Pro512, Pro514 \\
\hline celecoxib & Asn87, Lys511 & $2.38,1.82$ & Tyr91, Tyr475, Pro512, Pro514 \\
\hline
\end{tabular}

NA not available

benzene rings of compounds 62 and 63 were para-disubstituted. Last, one strong band at $835 \mathrm{~cm}^{-1}$ and another two bands at 765 and $695 \mathrm{~cm}^{-1}$ confirmed the existence of para-disubstituted and mono-substituted benzene rings in the structure of $\mathbf{6 1}$.

Analysis of the ${ }^{1} \mathrm{H}$ NMR spectra of compounds 61-63 (Table 6) revealed the existence of sydnone protons, styryl protons and benzene protons within the expected ranges when compared to the spectral data of the structurally-related analogues like styryl phenyl ketone [23], styryl naphthyl ketone [22] and mon-sydnone styryl ketones [13, 25]. Interestingly, the styryl protons of $\mathbf{6 2}$ and 63 appeared as doublets with coupling constants $\approx 16 \mathrm{~Hz}$ denoting the formation of trans conformers [23]. The splitting pattern of the styryl protons of $\mathbf{6 1}$ was not clear because they were overlapped with the aryl protons. However, the two bulky substituents favour the formation of trans isomer. Therefore, we surmise that the aldol condensation of the acetylated sydnones and the aldehyde sydnone derivative resulted in bis-sydnone trans-styryl ketones.

Additional structural elucidation was performed using 13C NMR spectroscopy (supplementary data S111, S115 and S119). The obtained spectra displayed three peaks in the carbonyl region. The first peak had an almost constant chemical shift about $174 \mathrm{ppm}$ in the three spectra and it was assigned to the carbonyl of the styryl ketone C14 [23]. The second peak at $168.92 \mathrm{ppm}$ was also stable and it was assigned to the sydnone carbonyl C5 because the chemical environment of this carbon atom was nearly identical in the three compounds. The third carbonyl peak at $\approx 166 \mathrm{ppm}$ was assigned to the sydnone carbonyl $\mathrm{C5}^{\prime}$ by a simple comparison with the acetylated sydnone counterparts $(\mathbf{4 9}, 50$ and $\mathbf{5 2})$. The sydnone carbon atom $\mathrm{C} 4$ appeared at $\approx 95 \mathrm{ppm}$ in the three spectra which was in accordance with the literature reported 


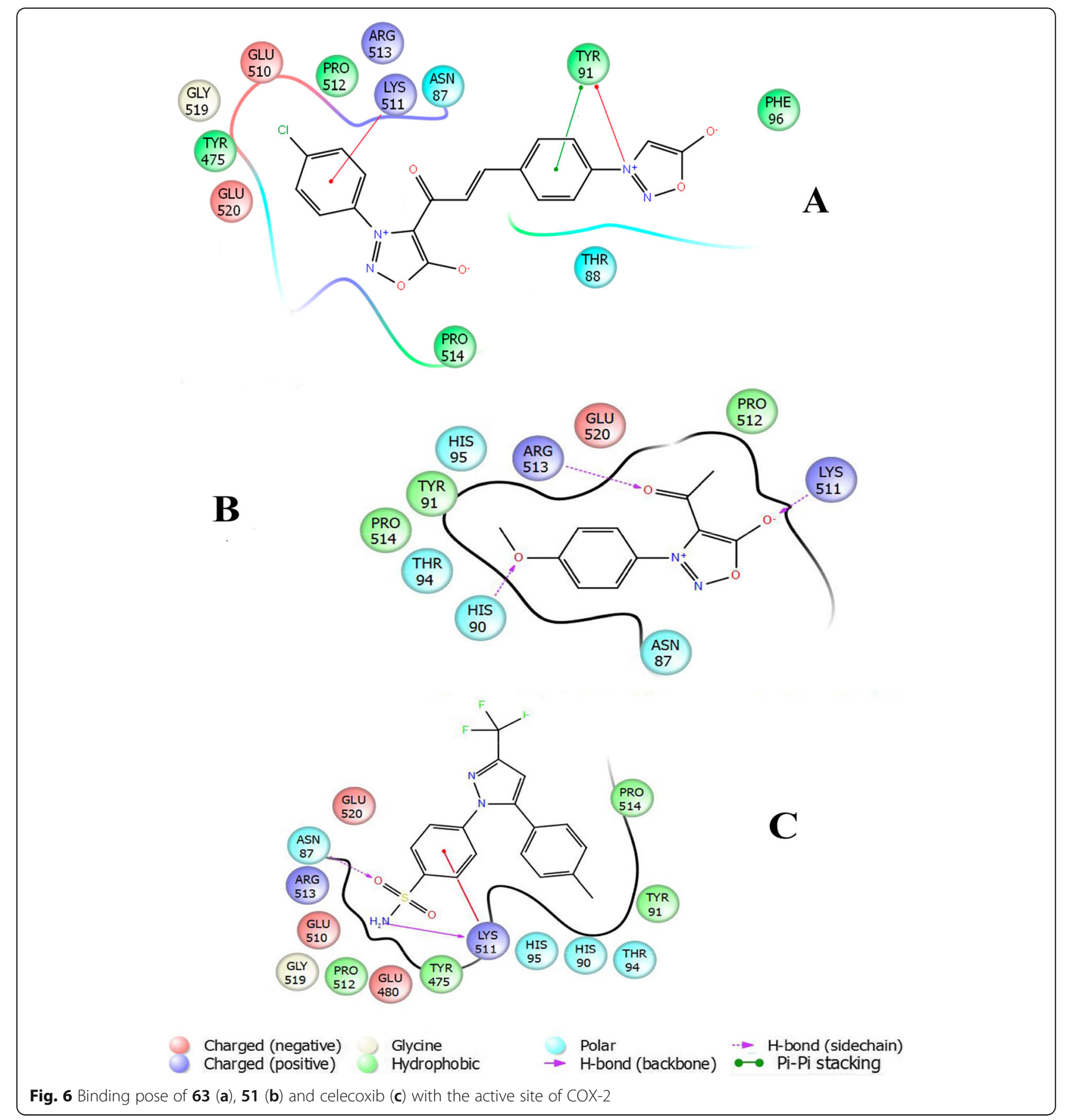

values for this sydnone characteristic peak. Similarly, the carbon atom C4' appeared deshielded at $\approx 108$ ppm because of the substituted acetyl group [26-30]. Interestingly, the chemical shifts of $\mathrm{C} 12$ and $\mathrm{C} 13$ of the styryl double bond were also nearly similar in the three compounds. The carbon $\mathrm{C} 12$ was the most deshielded because of the $\pi$ electron systems of the styryl and the benzene and it appeared at 142.5-141.5 ppm in the three spectra which came in agreement with the reported values for the carbon $\beta$ of other styryl ketone derivatives; $148-141 \mathrm{ppm}$. Moreover, the carbon atom C13 appeared at $\approx 124 \mathrm{ppm}$ which well matched the literature spectral chemical shifts of 125-121 ppm for the carbon $\alpha[13,23]$. The carbon atoms of the two benzene rings $\mathrm{C} 6-\mathrm{C} 11$ and $\mathrm{C}^{\prime}{ }^{\prime}-\mathrm{C} 11^{\prime}$ were assigned to the corresponding peaks by comparing the ${ }^{13} \mathrm{C}$ NMR spectra of 61, 62 and 63 with those of the corresponding acetylated analogues 49, 50 and $\mathbf{5 2}$. 


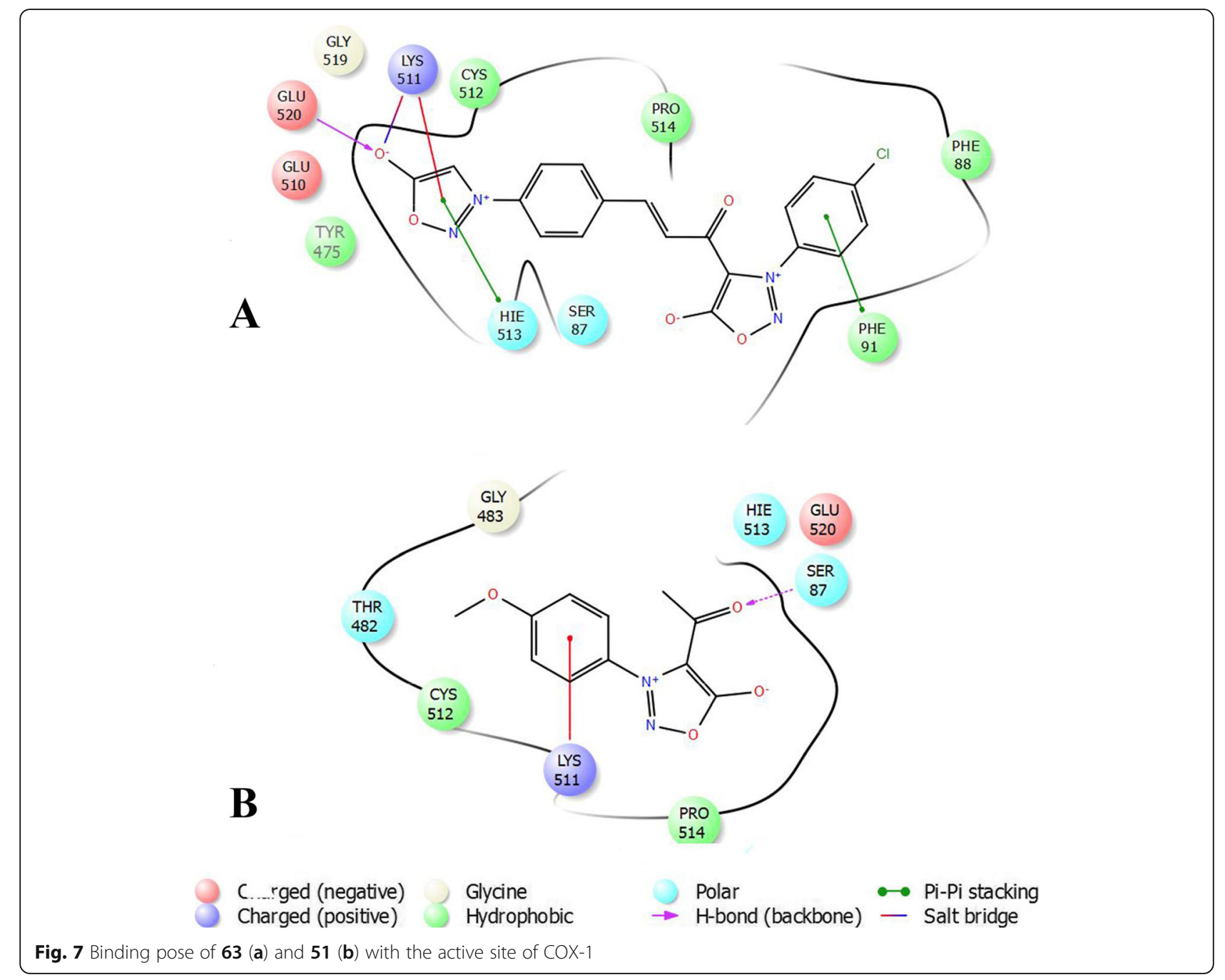

\section{In vitro cyclooxygenase inhibitory activity}

The anti-inflammatory activity of sydnone-based derivatives dates back to the 70s when researchers reported 3(2-arylthioethyl)sydnone and 3-(2-arylsulfoxyethyl)sydnone as potent anti-inflammatory drugs [6, 7]. Later, more experiments were undertaken to prepare other anti-inflammatory agents containing sydnone scaffolds like 4-(4-thiazolyl)sydnone [31]. However, studies on the anti-inflammatory activity of sydnones were conducted in vivo, and they were reported as anti-inflammatory, analgesic, antipyretic and anti-arthritis agents without exploring the effect of sydnones on the main enzyme involved in the inflammation process; cyclooxygenase $[32,33]$. Because the pharmaceutical inhibition of COX enzymes can provide relief from inflammation and pain, we initiated the current study to evaluate the inhibitory activity and the selectivity of various sydnone derivatives against both COX isoforms.

The outcomes of the present study showed that sydnone ring is not expected to form a general structure type for designing a potent COX inhibitor. On the contrary, the literature sources reported many sydnone derivatives as efficacious anti-inflammatory agents. A displayed in Fig. 8, a deep insight into structures I-IV which were delineated in the literature as effective antiarthritis and anti-edema agents $[6,31,33]$ concludes the fact that their bioactivity cannot be attributed to 3phenylsydnone. Other studies reported antiinflammatory effects of triheterocyclic thiazoles [34] and sulfoxide-containing compounds [34, 35]. Thereupon, thiazole, benzophenone oxime and sulfoxide are the main acting pharmacophores of $\mathbf{I}-\mathbf{I V}$. This discussion explains the reason for the moderate to low COX inhibitory activity of 37-58 which had the sydnone as the main scaffold.

Therefore, we attempted to improve the antiinflammatory properties of sydnones by joining two sydnone rings using an $\alpha, \beta$-unsaturated ketone linker (styryl ketone). The new compounds 61-63 were designed to have a common architecture and pharmacophoric 


\begin{tabular}{|c|c|c|c|c|}
\hline & \multicolumn{4}{|c|}{ Frequency $\left(v, \mathrm{~cm}^{-1}\right)$} \\
\hline & $61(\mathbf{R}=\mathbf{H}) *$ & $62\left(\mathrm{R}=\mathrm{CH}_{3}\right) * *$ & $63(\mathbf{R}=\mathbf{C l}) * * *$ & Reference values $^{1}$ \\
\hline \multicolumn{5}{|l|}{ C-H stretch } \\
\hline Sydnone & 3133 & 3171 & 3146 & $3150-3100$ \\
\hline Styryl & 3105 & 3114 & 3121 & $3095-3010$ \\
\hline Benzene & 3070 & 3091,3065 & 3075,3061 & $3100-3000$ \\
\hline \multicolumn{5}{|l|}{ CO stretch } \\
\hline Sydnone & 1778 & 1787 & 1777 & $1790-1750$ \\
\hline Sydnone & 1754 & 1765 & 1739 & $1790-1750$ \\
\hline styrylketone & 1655 & 1658 & 1659 & $1670-1600$ \\
\hline \multicolumn{5}{|l|}{$\mathrm{C}=\mathrm{C}$ stretch } \\
\hline Styryl & 1599 & 1605 & 1598 & $\approx 1600$ \\
\hline Benzene & $1588-1422$ & $1520-1425$ & $1520-1431$ & $1600-1450$ \\
\hline \multicolumn{5}{|l|}{ C-H out-of-plane bending } \\
\hline Styryl & 1030 & 1040 & 1033 & $1070-970$ \\
\hline Benzene & $835,765,695$ & 825 & 839 & $850-800$ \\
\hline & & & & $\approx 690$ \\
\hline & & & & $\approx 750$ \\
\hline
\end{tabular}

* Supplementary S109. ** Supplementary S113. *** Supplementary S117

a $[2,13,22,23,25]$

characters with some selective COX-2 inhibitors as highlighted in Fig. 9. As expected, the new molecules exhibited potent to moderate inhibitory activity at a concentration of $200 \mu \mathrm{M}$ against at least one of the COX isozymes. Noteworthy, we found that compound $\mathbf{6 3}$ selectively inhibited COX-2 with minimal effect on COX-1. Some studies had already demonstrated the anti-inflammatory activity of phenyl styryl ketone (chalcone). These researches showed that 3,4-dihydroxychalcone derivatives had a potent antiinflammatory activity due to 5-lipoxygenase and cyclooxygenase inhibition with $\mathrm{IC}_{50}$ values in the $\mu \mathrm{M}$ range [36]. Other researchers reported that some chalcone derivatives significantly reduced prostaglandin production in mouse peritoneal macrophages due to the inhibition of COX-2 synthesis. They demonstrated that chalcone derivatives did not affect cell viability indicating a low cellular toxicity [37]. More recently, Deshpande and his co-researchers reported the synthesis of sydnonyl styryl ketone as potential non-steroidal anti-inflammatory agents. They proved that these molecules had a topical and systematic anti-inflammatory activity with less ulcerogenic side effects. The most significant activity was associated with the existence of electron-withdrawing substituents at the para position of the benzene ring [13].

\section{Molecular docking analysis}

Cyclooxygenase (COX) or prostaglandin endoperoxide $\mathrm{H}$ synthase has two isozymes, commonly referred to as COX-1 and COX-2, each possessing different physiological effects. Mainly, COX-2 is highly expressed in stimulated macrophages, fibroblasts, osteoblasts, synoviocytes, endothelial cells, smooth muscle cells, central nervous system, cells of the female reproductive system and mast cells as a response to inflammatory mediators like LPS, IL-1, TNF $\alpha$ and INF $\gamma$. On the other hand, COX-1 is regularly present in most body tissues and is involved in normal homeostasis [38]. There is a high degree of amino-acid sequence similarity between both isozymes. The tertiary and quaternary structures of both COX isoforms are identical with some minor differences that are considered essential for selectively inhibiting either one. The COX molecule is a homodimer with a molecular weight of 67-72 kDa. The monomer of both isoforms consists of three distinct regions: The $\mathrm{N}$ terminal epidermal growth factor-like module (EGF) and the membrane binding domain (MBD) lying at the base of the third region which is the catalytic domain (CD). The CD contains peroxidase and COX active sites [39]. The $C D$ is the largest region, and its structure is virtually similar between COX-1 and COX-2 (RMS deviation of $0.4 \AA$ ). The residues Arg120, Tyr355 and Glu524 form 


\begin{tabular}{|c|c|c|c|c|c|}
\hline & & $\mathrm{H}$ & $\underbrace{12}_{9} \underbrace{H}_{13}$ & $\underbrace{+_{y^{\prime}}^{\prime}}_{\mathrm{O}^{\prime}}$ & \\
\hline \multicolumn{6}{|c|}{${ }^{1} \mathrm{H}$ NMR chemical shift ( $\delta$ ppm) } \\
\hline Molecular part & Proton & $\begin{array}{l}61 \\
(\mathbf{R}=\mathbf{H}) *\end{array}$ & $\left(\mathbf{R}=\mathbf{C H}_{3}\right) * *$ & $\mathbf{6 3}$ & Reference values ${ }^{1}$ \\
\hline Phenyl styryl ring & $\begin{array}{l}\text { C7-H, C11-H } \\
\text { C10-H, C8-H }\end{array}$ & $\begin{array}{l}8.04 \\
(\mathrm{~d}, \mathrm{~J}=8.51 \mathrm{~Hz}) \\
7.99 \\
(\mathrm{~d}, \mathrm{~J}=8.51 \mathrm{~Hz})\end{array}$ & $\begin{array}{l}8.19 \\
(\mathrm{~d}, \mathrm{~J}=8.51 \mathrm{~Hz}) \\
7.99 \\
(\mathrm{~d}, \mathrm{~J}=8.86 \mathrm{~Hz})\end{array}$ & $\begin{array}{l}8.05 \\
(d, J=8.86 \mathrm{~Hz}) \\
8.01 \\
(d, J=8.52 \mathrm{~Hz})\end{array}$ & $8.69-7.81\left(200 \mathrm{MHz}, \mathrm{CDCl}_{3}\right)$ \\
\hline Sydnone ring & $\mathrm{C} 4-\mathrm{H}$ & $7.85(\mathrm{~s})$ & $7.85(\mathrm{~s})$ & $7.85(\mathrm{~s})$ & - \\
\hline Styryl ketone & $\begin{array}{l}\mathrm{C} 12-\mathrm{H} \\
\mathrm{C} 13-\mathrm{H}\end{array}$ & $\begin{array}{l}7.81-7.74(\mathrm{~m}) \\
7.81-7.74(\mathrm{~m})\end{array}$ & $\begin{array}{l}7.83 \\
(\mathrm{~d}, \mathrm{~J}=16.01 \mathrm{~Hz}) \\
7.74 \\
(\mathrm{~d}, \mathrm{~J}=16.00 \mathrm{~Hz}) \\
\end{array}$ & $\begin{array}{l}7.84 \\
(d, J=16.01 \mathrm{~Hz}) \\
7.77 \\
(d, J=15.67 \mathrm{~Hz})\end{array}$ & $\begin{array}{l}\text { 7.85-7.75 }\left(300 \mathrm{MHz}, \mathrm{CDCl}_{3}\right) \\
\text { 7.64-7.45 }\left(300 \mathrm{MHz}, \mathrm{CDCl}_{3}\right)\end{array}$ \\
\hline $\begin{array}{l}\text { Phenyl ring at N3' } \\
\text { of the sydnone }\end{array}$ & $\begin{array}{l}\mathrm{C} 7^{\prime}-\mathrm{H}, \mathrm{C} 11^{\prime}-\mathrm{H} \\
\mathrm{C} 10^{\prime}-\mathrm{H}, \mathrm{C} 8^{\prime}-\mathrm{H} \\
\mathrm{R}\end{array}$ & $\begin{array}{l}7.81-7.74(\mathrm{~m}) \\
7.68 \\
(\mathrm{t}, \mathrm{J}=7.67 \mathrm{~Hz}) \\
7.81-7.74(\mathrm{~m})\end{array}$ & $\begin{array}{l}7.64 \\
(\mathrm{~d}, \mathrm{~J}=8.17 \mathrm{~Hz}) \\
7.48 \\
(\mathrm{~d}, \mathrm{~J}=8.18 \mathrm{~Hz}) \\
2,46\left(\mathrm{~s}, \mathrm{CH}_{3}\right)\end{array}$ & $\begin{array}{l}7.82 \\
(\mathrm{~d}, \mathrm{~J}=8.86 \mathrm{~Hz}) \\
7.79 \\
(\mathrm{~d}, \mathrm{~J}=8.85 \mathrm{~Hz}) \\
-\end{array}$ & $8.01-7.47\left(90 \mathrm{MHz}, \mathrm{CDCl}_{3}\right)$ \\
\hline
\end{tabular}

* Supplementary S110. ** Supplementary S114. *** Supplementary S118

a $[13,22,23,25-27]$

the entrance of the active site, while Tyr385 and Tyr348 lie at the apex of the active site and they are hydrogen bonded. The main differences between both active sites are at the positions 434, 513 and 523. In other words, Ile434 and Ile523 in COX-1 are replaced by valine residues in COX-2. The hydrophobic His513 in COX-1 was superseded by the positively charged arginine in COX-2. These differences resulted in altering the shape, size and the chemical environment of the active site of COX-2, and consequently, they are the key points of selective inhibitors [39, 40].

Binding energy $\Delta G_{\text {bind }}$ was widely used in the literature as a predictive tool to compare the potency of congeneric inhibitors against a specific enzyme. The free binding energy $\Delta G_{\text {bind }}$ was calculated from the formula:

$$
\Delta \mathrm{G}_{\text {bind }}=\Delta \mathrm{E}_{\mathrm{MM}}+\Delta \mathrm{G}_{\text {solv }}+\Delta \mathrm{G}_{\mathrm{SA}}
$$

where $\Delta E_{\mathrm{MM}}$ is the difference in energy between ligandenzyme complex and the sum of the energies of the ligand and free protein, $\Delta G_{\text {solv }}$ is the difference in the GBSA solvation energy between the complex and the free ligand and protein and $\Delta G_{\mathrm{SA}}$ is the difference in the surface area energy between the complex and the sum of the surface area energies for the ligand and free protein [41]. The linear relationship between the virtually estimated $\Delta G_{\text {bind }}$ and the experimental activity was well established for structurally similar data set. For example, Xie and his co-researchers reported a strong linear relationship $\left(r^{2}=0.87\right)$ between the experimental $\mathrm{IC}_{50}$ values of benzodiazepines and the calculated MM-GBSA of their complexes with gammaaminobutyric acid (GABA) receptor type A [42]. Moreover, MM-GBSA was $87-85 \%$ able to correctly rank the potency of checkpoint kinase 1 (CK1) inhibitors [16]. In the present study, it was found that the calculated binding energy values were able to explain $83 \%$ and $63 \%$ of the variation in the activity against $\mathrm{COX}-2$ and $\mathrm{COX}-1$, respectively. In other words, the in vitro COX-1/COX-2 inhibitory findings were well correlated with the in silico predicted binding poses of sydnones 37-63.

The residue 513 plays a paramount role in distinguishing the structural differences between the active sites of COX-1 and COX-2. Arginine forms a stable positive charge in the core of the COX-2 active site while histidine forms an exclusively hydrophobic pocket in COX-1 [43, 44]. The reason mentioned above illustrates one way in which some sydnone analogues of the present study selectively inhibit COX-2. For example, compound 63 was found to be the best selective COX-2 inhibitor in vitro. It exhibited many polar, positive or negative charged interactions with fewer hydrophobic interactions inside the entirely hydrophobic pocket of the COX-1 resulting in an 
<smiles>[R]Nc1nc(-c2c([O-])on[n+]2-c2ccc([R])cc2)cs1</smiles>

unstable complex with this enzyme (Fig. 7a). On the contrary, the sydnone ring had a positive charge interaction with Arg513 of COX-2 forming a tight enzyme-ligand complex (Fig. 6a and supplementary data S121). The same discussion is likewise with compound $\mathbf{5 1}$, which formed a positive charge interaction and a hydrogen bond with Arg513 of COX-2 (Fig. 6b and supplementary data S121), albeit it had a polar interaction with the hydrophobic histidine residue of COX-1 (Fig. 7b).
Cyclooxygenase enzymes were also known as membrane-bound proteins. They bind to the endoplasmic reticulum (ER) membrane through the interaction of the hydrophobic residues of MBD with one sheet of the lipid bilayer where they are processed and folded to become mature enzymes [45]. Apart from its role in protein-membrane binding, the MBD forms the route from which arachidonic acid (AA) enters the COX active site. The residues from 379 to 385 are the apex of the

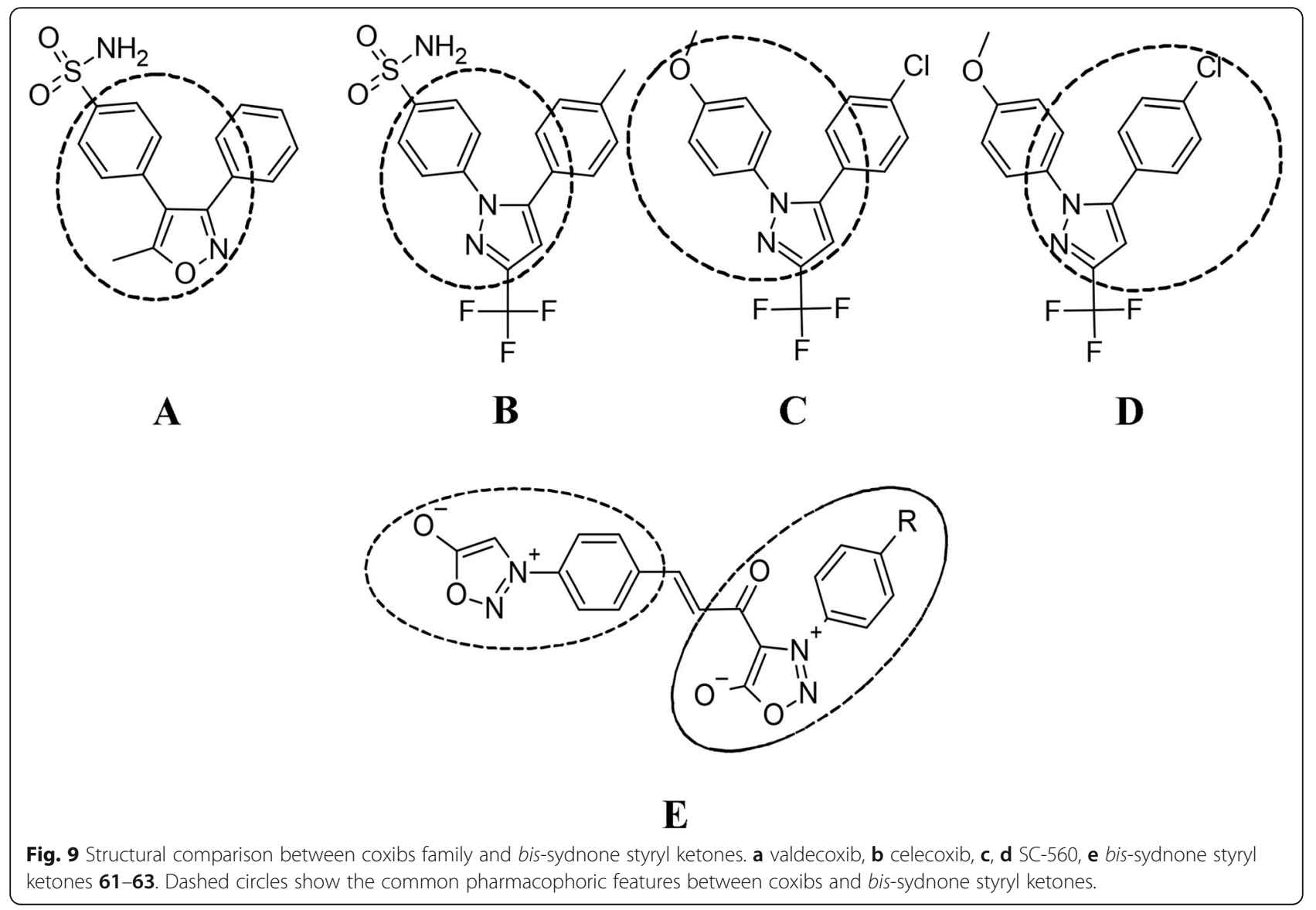


active site and have been known to form a proper positioning of AA [46]. Furthermore, Tyr355 lies on the opposite side of the mouth of the active site and controls the stereoselectivity of the inhibitors towards COX-2 [45]. The preceding discussion interprets the rationale for the high COX-1 inhibitory activity of compounds 37 , 39 and 41 which had hydrophobic interactions with Tyr355, Trp387 and Tyr385 blocking AA from producing cyclooxygenase product PGG2 (Fig. 10). In contrast, the sydnone and benzene rings in these three molecules

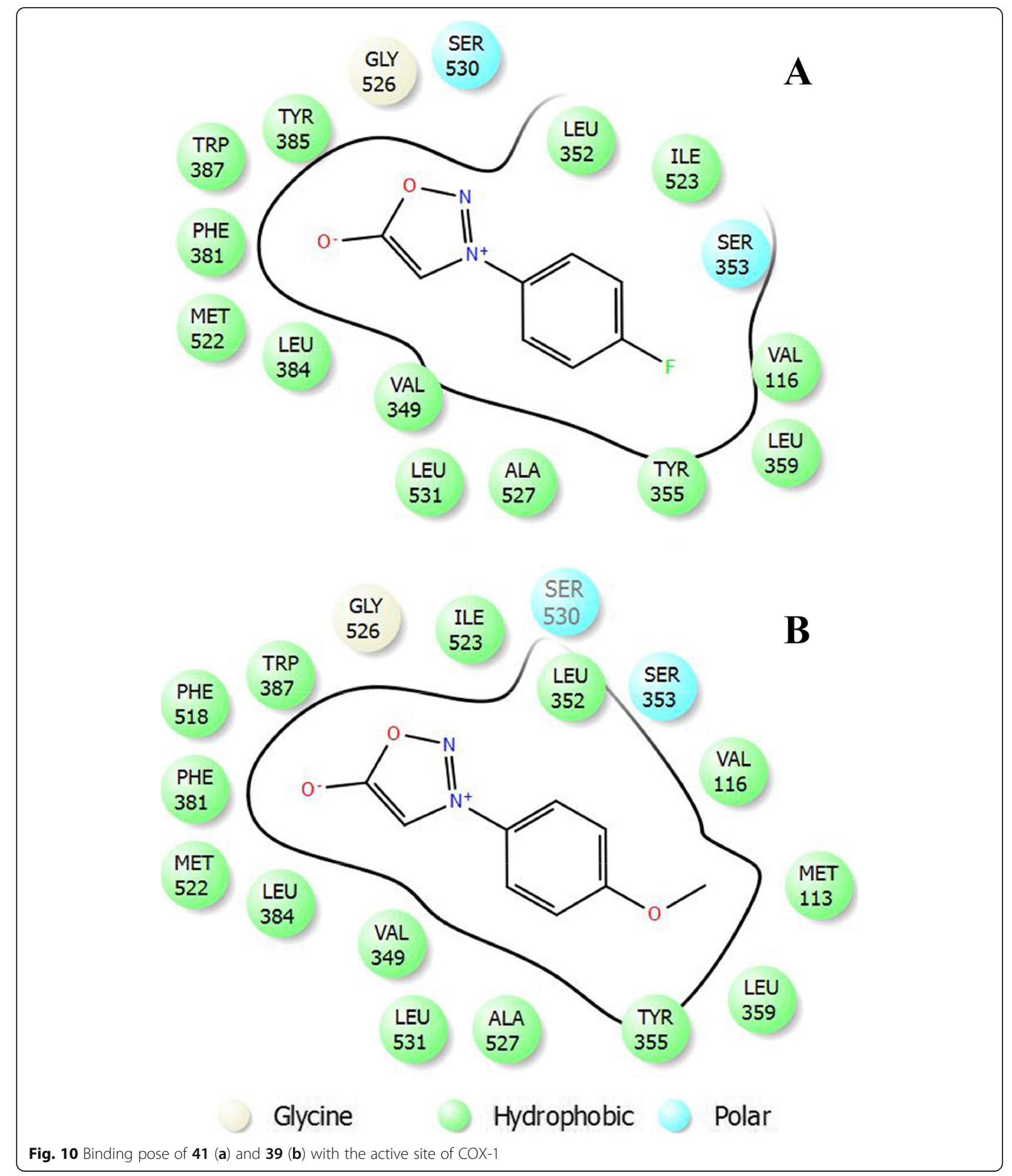


were involved in hydrophobic interactions with residues at the top of the COX-2 active site (Pro512, Pro514, etc.) rather than the apex which justifies their moderate COX-2 inhibitory activity.

\section{Conclusion}

In the present study, we described the synthesis of twenty-five sydnone-containing compounds including 3arylsydnones (37-48), 4-acetyl-3-arylsydnones (49-58) and trans bis-sydnone styryl ketones (61-63). The classical Aldol condensation was used to prepare bis-sydnone styryl ketones with a yield of $18-25 \%$. Compounds 61-63 containing two sydnone rings linked via a styryl ketone are three novel molecules reported herewith. The in vitro findings showed that neither 3-arylsydnone nor 4-acetyl-3-arylsydnone were a promising COX antagonist. Hybridization of sydnone with styryl ketone (chalcone) led to an improved COX inhibition. Compound 63 with a chlorine atom at the para position of the benzene ring inhibited more than $80 \%$ of COX-2 with a selectivity index above 2.42. The docking analysis showed that the binding hits of 63 (Tyr91, Tyr475, Pro512, Pro514) and 51 (Lys511, Arg513, Tyr91, Pro512, Pro514) are almost essential for COX-2 inhibitory activity. Of paramount, they form positively charged interaction and hydrogen bonding, respectively, with Arg513 of COX-2 active site. The general binding poses of $\mathbf{6 3}$ mimics that of celecoxib. Accordingly, bis-sydnone styryl ketones could be a potential lead for selective cyclooxygenase- 2 inhibitors and worth further chemical and biological consideration and investigations.

\section{Abbreviations}

SAR: Structure activity relationship; COX-1: Cyclooxygenase-1; COX-

2: Cyclooxygenase-2; TLC: Thin layer chromatography; TFAA: Trifluoroacetic anhydride; TMS: Tetramethylsilane; ESI: Electrospray ionisation; HRMS: Highresolution mass spectrometry; NMR: Nuclear magnetic resonance spectroscopy; FTIR: Fourier-transform infrared spectroscopy; SI-2: COC-2 selectivity index; SDF: Standard structure-data file; OPLS: Optimised potential for liquid simulations; PDB: Protein Data Bank; XP: Extra precision; MMGBSA: Molecular mechanics/generalised Born surface area;

LPS: Lipopolysaccharide; IL-1: Interleukin-1; TNFa: Tumor necrosis factor alpha; INFY: Interferon gamma; EGF: Epidermal growth factor;

MBD: Membrane binding domain; CD: Catalytic domain; ER: Endoplasmic reticulum; AA: Arachidonic acid

\section{Supplementary Information}

The online version contains supplementary material available at https://doi. org/10.1186/s43094-021-00216-4

\section{Additional file 1: FTIR, NMR and MS spectra of the synthesized} compounds along with 3D interaction diagram are available in the supplementary data. Spectrum 1. Infrared spectrum of $\mathbf{6}$ in $\mathrm{KBr}$ disc. Spectrum 2. ${ }^{1} \mathrm{H}$ NMR spectrum of $\mathbf{6}$ in DMSO- $d_{6}$. Spectrum 3. Infrared spectrum of $\mathbf{7}$ in $\mathrm{KBr}$ disc. Spectrum 4. ${ }^{1} \mathrm{H}$ NMR spectrum of $\mathbf{7}$ in DMSO$d_{6}$. Spectrum 5. Infrared spectrum of $\mathbf{8}$ in $\mathrm{KBr}$ disc. Spectrum 6. ${ }^{1} \mathrm{H}$ NMR spectrum of $\mathbf{8}$ in DMSO- $d_{6}$. Spectrum 7. Infrared spectrum of $\mathbf{9}$ in $\mathrm{KBr}$ disc. Spectrum 8. ${ }^{1} \mathrm{H}$ NMR spectrum of $\mathbf{9}$ in $\mathrm{DMSO}^{-} d_{6}$. Spectrum 9. Infrared spectrum of $\mathbf{1 0}$ in $\mathrm{KBr}$ disc. Spectrum 10. ${ }^{1} \mathrm{H}$ NMR spectrum of
10 in DMSO- $d_{6}$. Spectrum 11. Infrared spectrum of $\mathbf{1 2}$ in $\mathrm{KBr}$ disc. Spectrum 12. 'H NMR spectrum of 12 in DMSO- $d_{6}$. Spectrum 13. Infrared spectrum of $\mathbf{1 3}$ in $\mathrm{KBr}$ disc. Spectrum 14. ${ }^{1} \mathrm{H}$ NMR spectrum of $\mathbf{1 3}$ in $\mathrm{DMSO}_{-} d_{6}$. Spectrum 15. Infrared spectrum of $\mathbf{1 4}$ in $\mathrm{KBr}$ disc. Spectrum 16. ${ }^{1} \mathrm{H}$ NMR spectrum of 14 in DMSO- $d_{6}$. Spectrum 17. Infrared spectrum of $\mathbf{1 5}$ in $\mathrm{KBr}$ disc. Spectrum 18. ${ }^{1} \mathrm{H}$ NMR spectrum of 15 in $\mathrm{DMSO}_{-} d_{6}$. Spectrum 19. Infrared spectrum of $\mathbf{1 6}$ in $\mathrm{KBr}$ disc. Spectrum 20. ${ }^{1} H$ NMR spectrum of $\mathbf{1 6}$ in DMSO- $d_{6}$. Spectrum 21. Infrared spectrum of $\mathbf{1 7}$ in $\mathrm{KBr}$ disc. Spectrum 22. ${ }^{1} \mathrm{H}$ NMR spectrum of 17 in DMSO- $d_{6}$. Spectrum 23. Infrared spectrum of 22 in $\mathrm{KBr}$ disc. Spectrum 24. ${ }^{1} \mathrm{H}$ NMR spectrum of 22 in DMSO- $d_{6}$. Spectrum 25. Infrared spectrum of $\mathbf{2 3}$ in $\mathrm{KBr}$ disc. Spectrum 26. ${ }^{1} \mathrm{H}$ NMR spectrum of $\mathbf{2 3}$ in $\mathrm{DMSO}_{-} d_{6}$. Spectrum 27. Infrared spectrum of $\mathbf{2 4}$ in $\mathrm{KBr}$ disc. Spectrum 28. ${ }^{1} H$ NMR spectrum of 24 in DMSO- $d_{6}$. Spectrum 29. Infrared spectrum of $\mathbf{2 5}$ in $\mathrm{KBr}$ disc. Spectrum 30. ${ }^{1} \mathrm{H}$ NMR spectrum of $\mathbf{2 5}$ in DMSO- $d_{6}$. Spectrum 31. Infrared spectrum of $\mathbf{2 6}$ in $\mathrm{KBr}$ disc. Spectrum 32. ${ }^{1} H$ NMR spectrum of 26 in DMSO- $d_{6}$. Spectrum 33. Infrared spectrum of $\mathbf{2 7}$ in $\mathrm{KBr}$ disc. Spectrum 34. Infrared spectrum of $\mathbf{2 8}$ in $\mathrm{KBr}$ disc. Spectrum 35. Infrared spectrum of $\mathbf{2 9}$ in $\mathrm{KBr}$ disc. Spectrum 36. Infrared spectrum of $\mathbf{3 0}$ in $\mathrm{KBr}$ disc. Spectrum $\mathbf{3 7}$. Infrared spectrum of $\mathbf{3 1}$ in $\mathrm{KBr}$ disc. Spectrum 38. Infrared spectrum of $\mathbf{3 2}$ in $\mathrm{KBr}$ disc. Spectrum 39. Infrared spectrum of $\mathbf{3 3}$ in $\mathrm{KBr}$ disc. Spectrum 40. Infrared spectrum of $\mathbf{3 4}$ in $\mathrm{KBr}$ disc. Spectrum 41. Infrared spectrum of $\mathbf{3 5}$ in $\mathrm{KBr}$ disc. Spectrum 42. Infrared spectrum of $\mathbf{3 6}$ in $\mathrm{KBr}$ disc. Spectrum 43. Infrared spectrum of $\mathbf{3 7}$ in $\mathrm{KBr}$ disc Spectrum 44. ${ }^{1} \mathrm{H}$ NMR spectrum of 37 in $\mathrm{CDCl}_{3}$. Spectrum $45 .{ }^{13} \mathrm{C}$ NMR spectrum of $\mathbf{3 7}$ in $\mathrm{CDCl}_{3}$. Spectrum 46. Infrared spectrum of $\mathbf{3 8}$ in $\mathrm{KBr}$ disc. Spectrum 47. ${ }^{1} \mathrm{H}$ NMR spectrum of $\mathbf{3 8}$ in $\mathrm{CDCl}_{3}$. Spectrum 48. ${ }^{13} \mathrm{C}$ NMR spectrum of $\mathbf{3 8}$ in $\mathrm{CDCl}_{3}$. Spectrum 49. Infrared spectrum of $\mathbf{3 9}$ in $\mathrm{KBr}$ disc. Spectrum 50. ${ }^{1} \mathrm{H}$ NMR spectrum of $\mathbf{3 9}$ in $\mathrm{CDCl}_{3}$.

Spectrum 51. ${ }^{13} \mathrm{C}$ NMR spectrum of $\mathbf{3 9}$ in $\mathrm{CDCl}_{3}$. Spectrum 52. Infrared spectrum of $\mathbf{4 0}$ in $\mathrm{KBr}$ disc. Spectrum 53. ${ }^{1} \mathrm{H}$ NMR spectrum of $\mathbf{4 0}$ in $\mathrm{CDCl}_{3}$. Spectrum 54. ${ }^{13} \mathrm{C}$ NMR spectrum of $\mathbf{4 0}$ in $\mathrm{CDCl}_{3}$. Spectrum $\mathbf{5 5}$. Infrared spectrum of $\mathbf{4 1}$ in $\mathrm{KBr}$ disc. Spectrum 56. ${ }^{1} \mathrm{H}$ NMR spectrum of $\mathbf{4 1}$ in $\mathrm{CDCl}_{3}$. Spectrum 57. ${ }^{13} \mathrm{C}$ NMR spectrum of $\mathbf{4 1}$ in $\mathrm{CDCl}_{3}$. Spectrum 58. Infrared spectrum of 42 in $\mathrm{KBr}$ disc. Spectrum 59. ${ }^{1} \mathrm{H}$ NMR spectrum of $\mathbf{4 2}$ in DMSO- $d_{6}$. Spectrum $\mathbf{6 0} .{ }^{13} \mathrm{C}$ NMR spectrum of $\mathbf{4 2}$ in DMSO- $d_{6}$. Spectrum 61. Infrared spectrum of $\mathbf{4 3}$ in $\mathrm{KBr}$ disc. Spectrum 62. ${ }^{1} \mathrm{H}$ NMR spectrum of 43 in DMSO- $d_{6}$. Spectrum $63 .{ }^{13} \mathrm{C}$ NMR spectrum of $\mathbf{4 3}$ in DMSO- $d_{6}$. Spectrum 64. Infrared spectrum of 44 in $\mathrm{KBr}$ disc. Spectrum 65. ${ }^{1} \mathrm{H}$ NMR spectrum of $\mathbf{4 4}$ in DMSO- $d_{6}$. Spectrum 66. ${ }^{13} \mathrm{C}$ NMR spectrum of $\mathbf{4 4}$ in DMSO- $d_{6}$. Spectrum 67. Infrared spectrum of $\mathbf{4 5}$ in $\mathrm{KBr}$ disc. Spectrum 68. ${ }^{1} \mathrm{H}$ NMR spectrum of 45 in DMSO- $d_{6}$. Spectrum 69. ${ }^{13} \mathrm{C}$ NMR spectrum of $\mathbf{4 5}$ in DMSO- $d_{6}$. Spectrum 70. Infrared spectrum of 46 in $\mathrm{KBr}$ disc. Spectrum 71. ${ }^{1} \mathrm{H}$ NMR spectrum of $\mathbf{4 6}$ in DMSO- $d_{6}$. Spectrum 72. ${ }^{13} \mathrm{C}$ NMR spectrum of 46 in $\mathrm{DMSO}_{-} d_{6}$. Spectrum 73. Infrared spectrum of $\mathbf{4 7}$ in $\mathrm{KBr}$ disc. Spectrum 74. ${ }^{1} \mathrm{H}$ NMR spectrum of 47 in DMSO- $d_{6}$. Spectrum 75. ${ }^{13} \mathrm{C}$ NMR spectrum of $\mathbf{4 7}$ in DMSO- $d_{6}$. Spectrum 76. Infrared spectrum of 48 in $\mathrm{KBr}$ disc. Spectrum 77. ${ }^{1} \mathrm{H}$ NMR spectrum of $\mathbf{4 8}$ in DMSO- $d_{6}$. Spectrum 78. ${ }^{13} \mathrm{C}$ NMR spectrum of $\mathbf{4 8}$ in DMSO- $d_{6}$. Spectrum 79. Infrared spectrum of $\mathbf{4 9}$ in $\mathrm{KBr}$ disc. Spectrum 80. ${ }^{1} \mathrm{H}$ NMR spectrum of 49 in $\mathrm{CDCl}_{3}$. Spectrum 81. ${ }^{13} \mathrm{C}$ NMR spectrum of $\mathbf{4 9}$ in $\mathrm{CDCl}_{3}$. Spectrum 82. Infrared spectrum of $\mathbf{5 0}$ in $\mathrm{KBr}$ disc. Spectrum 83. ${ }^{1} \mathrm{H}$ NMR spectrum of $\mathbf{5 0}$ in $\mathrm{CDCl}_{3}$. Spectrum $\mathbf{8 4} .{ }^{13} \mathrm{C}$ NMR spectrum of $\mathbf{5 0}$ in $\mathrm{CDCl}_{3}$. Spectrum 85. Infrared spectrum of $\mathbf{5 1}$ in $\mathrm{KBr}$ disc. Spectrum 86. ${ }^{1} \mathrm{H}$ NMR spectrum of $\mathbf{5 1}$ in $\mathrm{CDCl}_{3}$. Spectrum 87. ${ }^{13} \mathrm{C}$ NMR spectrum of $\mathbf{5 1}$ in $\mathrm{CDCl}_{3}$. Spectrum 88. Infrared spectrum of $\mathbf{5 2}$ in $\mathrm{KBr}$ disc. Spectrum 89. ${ }^{1} \mathrm{H}$ NMR spectrum of $\mathbf{5 2}$ in $\mathrm{CDCl}_{3}$. Spectrum $\mathbf{9 0} .{ }^{13} \mathrm{C}$ NMR spectrum of $\mathbf{5 2}$ in $\mathrm{CDCl}_{3}$. Spectrum 91. Infrared spectrum of $\mathbf{5 3}$ in $\mathrm{KBr}$ disc. Spectrum 92. ${ }^{1} \mathrm{H}$ NMR spectrum of $\mathbf{5 3}$ in $\mathrm{CDCl}_{3}$. Spectrum 93. ${ }^{13} \mathrm{C}$ NMR spectrum of $\mathbf{5 3}$ in $\mathrm{CDCl}_{3}$. Spectrum 94. Infrared spectrum of $\mathbf{5 4}$ in $\mathrm{KBr}$ disc. Spectrum 95. ${ }^{1} \mathrm{H}$ NMR spectrum of $\mathbf{5 4}$ in DMSO- $d_{6}$. Spectrum 96. ${ }^{13} \mathrm{C}$ NMR spectrum of $\mathbf{5 4}$ in DMSO- $d_{6}$. Spectrum 97. Infrared spectrum of $\mathbf{5 5}$ in $\mathrm{KBr}$ disc. Spectrum 98. ${ }^{1} \mathrm{H}$ NMR spectrum of $\mathbf{5 5}$ in DMSO- $d_{6}$. Spectrum 99. ${ }^{13} \mathrm{C}$ NMR spectrum of $\mathbf{5 5}$ in DMSO- $d_{6}$. Spectrum 100. Infrared spectrum of $\mathbf{5 6} \mathrm{in} \mathrm{KBr}$ disc. Spectrum 101. ${ }^{1} \mathrm{H}$ NMR spectrum of $\mathbf{5 6}$ in DMSO- $d_{6}$. Spectrum 102. ${ }^{13} \mathrm{C}$ NMR spectrum of $\mathbf{5 6}$ in DMSO- $d_{6}$. Spectrum 103. Infrared spectrum of $\mathbf{5 7}$ in $\mathrm{KBr}$ disc. Spectrum 104. 'H NMR spectrum of $\mathbf{5 7}$ in DMSO- $d_{6}$. Spectrum 105. 
${ }^{13} \mathrm{C}$ NMR spectrum of $\mathbf{5 7}$ in DMSO- $d_{6}$. Spectrum 106. Infrared spectrum of $\mathbf{5 8}$ in $\mathrm{KBr}$ disc. Spectrum 107. ${ }^{1} \mathrm{H}$ NMR spectrum of $\mathbf{5 8}$ in DMSO- $d_{6}$. Spectrum 108. ${ }^{13} \mathrm{C}$ NMR spectrum of $\mathbf{5 8}$ in DMSO- $d_{6}$. Spectrum 109. Infrared spectrum of $\mathbf{6 1} \mathrm{in} \mathrm{KBr}$ disc. Spectrum 110. ${ }^{1} \mathrm{H}$ NMR spectrum of 61 in DMSO- $d_{6}$. Spectrum 111. ${ }^{13} \mathrm{C}$ NMR spectrum of $\mathbf{6 1}$ in DMSO- $d_{6}$. Spectrum 112. High resolution mass spectrum (HRMS) of $\mathbf{6 1}$. Spectrum 113. Infrared spectrum of 62 in $\mathrm{KBr}$ disc. Spectrum 114. ${ }^{1} \mathrm{H}$ NMR spectrum of $\mathbf{6 2}$ in DMSO- $d_{6}$. Spectrum $\mathbf{1 1 5} .{ }^{13} \mathrm{C}$ NMR spectrum of $\mathbf{6 2}$ in DMSO- $d_{6}$. Spectrum 116. High resolution mass spectrum (HRMS) of $\mathbf{6 2}$. Spectrum 117. Infrared spectrum of $\mathbf{6 3}$ in $\mathrm{KBr}$ disc. Spectrum 118. ${ }^{1} \mathrm{H}$ NMR spectrum of $\mathbf{6 3}$ in DMSO- $d_{6}$. Spectrum 119. ${ }^{13} \mathrm{C}$ NMR spectrum of 63 in DMSO- $d_{6}$. Spectrum 120. High resolution mass spectrum (HRMS) of 63. Supplementary S121. Docking results of compounds 63 (green), $\mathbf{5 1}$ (red) and celecoxib (blue) in the active pocket of COX-2 (pink) and their 3D alignment based on the docked conformations. Ligands are presented as ball and sticks, active pocket is presented as a surface, and important interacting residues are presented as yellow lines.

\section{Acknowledgements}

The authors would like to thank the National University of Singapore (NUS) for providing the mass spectroscopy service. We are also grateful to the Malaysia Genome Institute (MGl) for the NMR spectroscopy.

\section{Authors' contributions}

A.M.A. conception and design of the work, data analysis and interpretation and manuscript preparation; M.T. and N.I.N.Y. funding acquisition and project administration; M.A. manuscript preparation and revision. All authors have read and approved the manuscript.

\section{Funding}

The work was financially sponsored by the Ministry of Higher Education Malaysia through the Fundamental Research Grant Scheme (FRGS No. 130031-0272). FRGS grant funding covered all research requirements including chemical and material supplies, professional services such as MS and NMR fees, transportation costs, wages, maintenance.

\section{Availability of data and materials}

All data and materials should be available upon request.

\section{Declarations}

Ethics approval and consent to participate

No applicable.

\section{Consent for publication}

No applicable.

\section{Competing interests}

The authors declare that they have no competing interest.

\section{Author details}

'Pharmaceutical Chemistry Department, Faculty of Pharmacy, International Islamic University Malaysia, 25200 Kuantan, Pahang, Malaysia. ²Pharmaceutical Technology Department, Faculty of Pharmacy, International Islamic University Malaysia, 25200 Kuantan, Pahang, Malaysia.

Received: 28 October 2020 Accepted: 23 February 2021 Published online: 09 March 2021

\section{References}

1. Chandrasekhar R, Nanjan MJ (2012) Sydnones: a brief review. Mini Rev Med Chem 12(13):359-1365

2. Browne DL, Harrity JPA (2010) Recent developments in the chemistry of sydnones. Tetrahedron 66(3):553-568

3. Abdualkader AM, Taher M, Nik Idirs NY (2017) Mesoionic sydnone: a review in their chemical and biological properties. Int J Phar Pharm Sci 9(8):1-9

4. Asundaria ST, Patel KC (2012) Synthesis, characterization and antimicrobial activity of thiazole, benzothiazole and pyrimidine derivatives bearing sydnone moieties. Pharm Chem J 45(12):725-731
5. Asundaria ST, Patel KC (2013) Synthesis, characterization, and antimicrobial evolution of bissydnone based on sulfonamide derivatives. J Heterocycl Chem 50(S1):E136-E141

6. Hill JB, Ray RE, Wanger RH, Aspinall RL (1975) Antiinflammatory sydnones. 2. J Med Chem 18(1):50-53

7. Wagner RH, Hill JB (1974) Antiinflammatory sydnones. 1. J Med Chem 17(12):1337-1338

8. Patel YM, Patel KC (2015) Synthesis and biological evaluation of new sydnone based derivatives. J Saudi Chem Soc 19(2):193-199

9. Dunkley CS, Thoman CJ (2003) Synthesis and biological evaluation of a novel phenyl substituted sydnone series as potential antitumor agents. Bioorg Med Chem Lett 13(17):2899-2901

10. Butković K, Marinić Z, Šindler-Kulyk M (2011) Synthesis of 3-(ostilbenyl)sydnone and 3-(o-stilbenyl)-4-substituted-sydnone derivatives and their antitumor evaluation. Arkivoc 10(10):1-15

11. Preston PN, Turnbull K (1977) Approaches to the synthesis of compounds containing fused mesoionic rings. J Chem Soc Perkin Trans 10:1229-1233

12. Greco CV, Tobias J, Kier LB (1967) Acylation of 3-phenylsydnone with carboxylic acids and phosphorus pentoxide. J Heterocycl Chem 4(1):160162

13. Deshpande SR, Pai KV (2012) Fluorine bearing sydnones with styryl ketone group: synthesis and their possible analgesic and anti-inflammatory activities. Enzyme Inhib Med Chem 27(2):241-248

14. Dadashpour S, Tuylu KT, Unsal TO, Ozadali K, Irannejad H, Emami S (2015) Design, synthesis and in vitro study of 5,6-diaryl-1,2,4-triazine-3-ylthioacetate derivatives as COX-2 and $\beta$-amyloid aggregation inhibitors. Arch Pharm 348(3):179-187

15. Harder E, Damm W, Maple J, Wu C, Reboul M, Xiang JY, Lupyan D, Dahlgren MK, Kaus J, Cerutti DS, Krilov G, Jorgensen WL, Abel R, Friesner RA (2016) OPLS3: a force field providing broad coverage of drug-like small molecules and proteins. J Chem Theory Comput 12(1):281-296

16. Du J, Sun H, Xi L, Li J, Yang Y, Liu H, Yao X (2011) Molecular modeling study of checkpoint kinase 1 inhibitors by multiple docking strategies and prime/ MM-GBSA calculation. J Comput Chem 32(13):2800-2809

17. Friesner RA, Murphy RB, Repasky MP, Frye LL, Greenwood JR, Halgren TA, Sanschagrin PC, Mainz DT (2006) Extra precision glide: docking and scoring incorporating a model of hydrophobic enclosure for protein-ligand complexes. J Med Chem 49(21):6177-6196

18. Li J, Abel R, Zhu K, Cao Y, Zhao S, Friesner RA (2011) The VSGB 2.0 model: a next generation energy model for high resolution protein structure modeling. Proteins 79(10):2794-2812

19. Christian B, Thorsten JM, Ronald S, Annette H, Lars Z, Per-Johan J, Klaus S, Gerd G (2006) Inhibition of prostaglandin E2 synthesis by SC-560 is independent of cyclooxygenase 1 inhibition. FASEB J 20(9):1352-1360

20. Rosenstock M, Danon A, Rimon G (1999) PGHS-2 inhibitors, NS-398 and DuP-697, attenuate the inhibition of PGHS-1 by aspirin and indomethacin without altering its activity. Biochim Biophys Acta Mol Cell Biol Lipids 1440(1):127-137

21. Smith MB (2019) March's advanced organic chemistry: reactions, mechanisms, and structure, 8th edn. Wiley, New Jersey

22. Thirunarayanan $G$ (2007) Synthesis, characterization and spectral correlations in substituted styryl 6-methyl-2-naphthyl ketones. Indian J Chem Sect B Org Med Chem 46(9):1511-1517

23. Thirunarayanan G, Vanangamudi G (2014) Synthesis, spectral studies, antimicrobial and insect antifeedant activities of some substituted styryl 4'fluorophenyl ketones. Arab J Chem 7(6):1055-1064

24. Deshpande SR, Pai KV, Pai SR (2011) Design and synthesis of certain mesoionic sydnonyl styrylketones as potential nonsteroidal antiinflammatory agents. Arzneimittel-Forschung/Drug Res 61(3):180-185

25. Deshpande SR, Pai KV (2010) Synthesis, antibacterial and analgesic activities of 4- [1-axo- 3-(substituted aryl) -2-propenyl]- 3-(4-methyl phenyl) sydnones. J Chem 7(1):59-64

26. Azarifar D, Bosra HG, Tajbaksh M (2007) 1,3-Dibromo-5,5-dimethylhydantoin $(\mathrm{DBH})$ as an efficient promoter for acetylation of 3-arylsydnones in the presence of acetic anhydride under neutral conditions. J Heterocycl Chem 44(2):467-469

27. Azarifar D, Bosra HG (2006) Catalytic activity of 1,3-dibromo-5,5dimethylhydantoin $(\mathrm{DBH})$ in the one-pot transformation of $\mathrm{N}$-arylglycines to $\mathrm{N}$-arylsydnones in the presence of NaNO2/Ac2O under neutral conditions: Subsequent bromination of these sydnones to their 4-bromo derivatives. Synthesis (Stuttg) 7:1123-1126 
28. Balaguer A, Selhorst R, Turnbull K (2013) Metal triflate-catalyzed friedel-crafts acetylation of 3-phenylsydnon. Synth Commun 43(12):1626-1632

29. Balaguer AM, Rumple AC, Turnbull K (2014) Bismuth triflate catalyzed Friedel-crafts acylation of 3-Phenyl- and 3-(2-carboalkoxyphenyl)sydnones under microwave irradiation. Org Prep Proced Int 46(4):363-369

30. Mahoney J, Turnbull K, Cubberley M (2012) Bismuth triflate-catalyzed friedelcrafts acylations of sydnones. Synth Commun 42(21):3220-3229

31. Upadhya KG, Badami BV, Puranik GS, Biradar VN, Nanjappa S (1980) Synthesis and antiinflammatory activity of 3-substituted 4-(4'-thiazolyl)sydnones. Arch Pharm 313(8):684-688

32. Satyanarayana K, Deshpande SR, Subba RB, Rao MN (2002) Synthesis and nitric oxide donor activity of phenylsydnones. Indian Drugs 39(11):578-582

33. Kamble RR, Belgur SS, Aladkatti R, Khazi IA (2009) Synthesis and evaluation of benzophenone oximes derivatized with sydnone as inhibitors of secretory phospholipase A2 with anti-inflammatory activity. Chem Pharm Bull 57(1):16-21

34. Steinberg A (1967) The employment of dimethyl sulfoxide as an antiinflammatory agent and steroid-transporter in diversified clinical diseases. Ann NY Acad Sci 141(1):532-550

35. Unnikrishnan MK, Rao MNA (1990) Antiinflammatory activity of methionine, methionine sulfoxide and methionine sulfone. Agents Actions 31(1):110-112

36. Sogawa S, Nihro Y, Ueda H, Izumi A, Miki T, Matsumoto H, Satoh T (1993) 3,4-Dihydroxychalcones as potent 5-lipoxygenase and cyclooxygenase inhibitors. J Med Chem 36(24):3904-3909

37. Herencia F, Ferrándiz ML, Ubeda A, Guillén I, Dominguez JN, Charris JE, Lobo GM, Alcaraz MJ (1999) Novel anti-inflammatory chalcone derivatives inhibit the induction of nitric oxide synthase and cyclooxygenase- 2 in mouse peritoneal macrophages. FEBS Lett 453(1-2):129-134

38. Herschman HR (1996) Prostaglandin synthase 2. Biochim Biophys Acta Lipids Lipid Metab 1299(1):125-140

39. Luong C, Miller A, Barnett J, Chow J, Ramesha C, Browner MF (1996) Flexibility of the NSAID binding site in the structure of human cyclooxygenase-2. Nat Struct Biol 3(11):927-933

40. Rimon G, Sidhu RS, Lauver DA, Lee JY, Sharma NP, Yuan C, Frieler RA, Trievel RC, Lucchesi BR, Smith WL (2010) Coxibs interfere with the action of aspirin by binding tightly to one monomer of cyclooxygenase-1. Proc Nat Acad Sci USA 107(1):28-33

41. Lyne PD, Lamb ML, Saeh JC (2006) Accurate prediction of the relative potencies of members of a series of kinase inhibitors using molecular docking and MM-GBSA scoring. J Med Chem 49(16):4805-4808

42. Xie HB, Sha Y, Wang J, Cheng MS (2013) Some insights into the binding mechanism of the GABAA receptor: a combined docking and MM-GBSA study. J Mol Model 19(12):5489-5500

43. Ferreira LG, Dos Santos RN, Oliva G, Andricopulo AD (2015) Molecular docking and structure-based drug design strategies. Molecules 20(7): 13384-13421

44. Alanazi AM, El-Azab AS, Al-Suwaidan IA, EITahir K, Asiri YA, Abdel-Aziz NI, Abdel-Aziz AAM (2015) Structure-based design of phthalimide derivatives as potential cyclooxygenase-2 (COX-2) inhibitors: anti-inflammatory and analgesic activities. Eur J Med Chem 92:115-123

45. Smith WL, Garavito RM, DeWitt DL (1996) Prostaglandin endoperoxide H synthases (cyclooxygenases)-1 and -2. J Biol Chem 271(52):33157-33160

46. Picot D, Loll PJ, Garavito RM (1994) The X-ray crystal structure of the membrane protein prostaglandin $\mathrm{H} 2$ synthase-1. Nature 367:243-249

\section{Publisher's Note}

Springer Nature remains neutral with regard to jurisdictional claims in published maps and institutional affiliations.

\section{Submit your manuscript to a SpringerOpen ${ }^{\circ}$ journal and benefit from:}

- Convenient online submission

- Rigorous peer review

- Open access: articles freely available online

- High visibility within the field

- Retaining the copyright to your article

Submit your next manuscript at $\boldsymbol{\nabla}$ springeropen.com 